\title{
Remarks on a class of renormalizable interpolating gauges
}

\author{
D. Dudal $^{a *}$, J.A. Gracey ${ }^{b \dagger}$, V.E.R. Lemes ${ }^{c \ddagger}$, R.F. Sobreiro ${ }^{c \S}$ \\ S.P. Sorella ${ }^{\mathbb{f} * *}$, R. Thibes ${ }^{c \dagger \dagger}$, H. Verschelde ${ }^{a \ddagger \ddagger}$ \\ a Ghent University \\ Department of Mathematical Physics and Astronomy \\ Krijgslaan 281-S9, B-9000 Gent, Belgium \\ ${ }^{b}$ Theoretical Physics Division \\ Department of Mathematical Sciences \\ University of Liverpool \\ P.O. Box 147, Liverpool, L69 3BX, United Kingdom \\ c UERJ, Universidade do Estado do Rio de Janeiro \\ Rua São Francisco Xavier 524, 20550-013 Maracanã \\ Rio de Janeiro, Brasil
}

\begin{abstract}
A class of covariant gauges allowing one to interpolate between the Landau, the maximal Abelian, the linear covariant and the Curci-Ferrari gauges is discussed. Multiplicative renormalizability is proven to all orders by means of algebraic renormalization. All one-loop anomalous dimensions of the fields and gauge parameters are explicitly evaluated in the $\overline{\mathrm{MS}}$ scheme.
\end{abstract}

\footnotetext{
${ }^{*}$ Research Assistant of The Fund For Scientific Research-Flanders, Belgium.

†jag@amtp.liv.ac.uk

${ }^{\ddagger}$ vitor@dft.if.uerj.br

${ }^{\S}$ sobreiro@uerj.br

`sorella@uerj.br

"Work supported by FAPERJ, Fundação de Amparo à Pesquisa do Estado do Rio de Janeiro, under the program Cientista do Nosso Estado, E-26/151.947/2004.

**sorella@uerj.br

${ }^{\dagger \dagger}$ thibes@dft.if.uerj.br

${ }^{\ddagger}$ david.dudal@ugent.be, henri.verschelde@ugent.be
} 


\section{Introduction.}

Interpolating gauges have been proven to be a powerful tool to achieve a better understanding of the behavior of the Green's functions in different gauges. Let us quote, for instance, the interpolating gauge introduced in [1], allowing one to connect the Landau and the light cone gauges, thereby providing a useful framework to investigate Yang-Mills theories in noncovariant gauges. Another example is provided by the interpolating gauge proposed in [2, 3], which connects the Landau and the Coulomb gauges. More recently, a class of covariant gauges interpolating between the Landau and the maximal Abelian gauge has been introduced in [4] in order to investigate the properties of the vacuum energy of Yang-Mills theories due to dimension two gauge condensates. More precisely, it has been possible to show that the vacuum energy obtained in the maximal Abelian gauge, due to the condensate $\left\langle\frac{A_{\mu}^{a} A_{\mu}^{a}}{2}+\alpha \bar{c}^{a} c^{a}\right\rangle$, is the same as the vacuum energy obtained in the Landau gauge, due to the condensate* $\left\langle\frac{A_{\mu}^{A} A_{\mu}^{A}}{2}\right\rangle$. We recall that a nonvanishing condensate $\left\langle\frac{A_{\mu}^{a} A_{\mu}^{a}}{2}+\alpha \bar{c}^{a} c^{a}\right\rangle$ in the maximal Abelian gauge results in a dynamical mass generation for the off-diagonal gluons. This can be seen as evidence for the Abelian dominance hypothesis, implying that Yang-Mills theories in the low energy region should be described by an effective abelian theory, according to the dual superconductivity mechanism for color confinement.

In this work we introduce a class of covariant gauges allowing one to interpolate between the Landau gauge, the maximal Abelian gauge, the linear covariant gauges and the Curci-Ferrari gauge. It is worth remarking that in all these gauges a nonvanishing dimension two condensate has emerged (see [4, 5] and references therein), so that this generalized interpolating gauge might be useful for further investigation of these condensates.

The work is organized as follows. In Sect. 2 we introduce the interpolating gauge fixing. Since the maximal Abelian gauge requires a splitting of the $S U(N)$ color index $A=(i, a)$, corresponding to the $N-1$ diagonal generators of the Cartan subalgebra of $S U(N)$, labeled by the index $i$, and to the $N(N-1)$ off-diagonal generators, labeled by $a$, it turns out that the gauge-fixing term contains six independent gauge parameters. In Sect. 3 we discuss the various limits for those gauge parameters, allowing one to recover the linear covariant gauges, the Landau gauge, the maximal Abelian gauge and the Curci-Ferrari gauge. In Sect. 4 we prove the all orders multiplicative renormalizability by means of the algebraic renormalization procedure. In Sect. 5 we present the explicit one-loop calculation of the anomalous dimensions of all fields and parameters. These reduce to the already known values in the various limits for the interpolating gauge parameters. In particular, as a useful check, it is verified that the beta function of the gauge coupling is independent of all the gauge parameters. In the concluding section we underline a potential application of the interpolating gauge for lattice simulations. Some technical details are collected in Appendix A.

\footnotetext{
${ }^{*}$ In the case of the maximal Abelian gauge, the index $a$ refers only to the $N(N-1)$ off-diagonal generators of $S U(N)$, while the index $A$ runs from 1 to $N^{2}-1$.
} 


\section{The interpolating gauge fixing term.}

Let $A_{\mu}$ be the Lie algebra valued connection for the gauge group $S U(N)$, whose generators $T^{A}$, satisfying $\left[T^{A}, T^{B}\right]=i f^{A B C} T^{C}$, are chosen to be hermitean and to obey the orthonormality condition $\operatorname{Tr}\left(T^{A} T^{B}\right)=T_{F} \delta^{A B}$, with $A, B, C=1, \ldots,\left(N^{2}-1\right)$. As needed in order to discuss the maximal Abelian gauge, we decompose the gauge field into off-diagonal and diagonal components, namely

$$
A_{\mu}=A_{\mu}^{A} T^{A}=A_{\mu}^{a} T^{a}+A_{\mu}^{i} T^{i},
$$

where the indices $i, j, k$ label the $N-1$ generators of the Cartan subalgebra. The remaining $N(N-1)$ off-diagonal generators will be labelled by the rest of the small Latin indices. For later use, we recall the Jacobi identity

$$
f^{A B C} f^{C D E}+f^{A D C} f^{C E B}+f^{A E C} f^{C B D}=0,
$$

from which it can be deduced that

$$
\begin{aligned}
f^{a b i} f^{b j c}+f^{a b j} f^{b c i} & =0, \\
f^{a b c} f^{b d i}+f^{a b d} f^{b i c}+f^{a b i} f^{b c d} & =0, \\
f^{a b c} f^{c d e}+f^{a b i} f^{i d e}+f^{a d c} f^{c e b}+f^{a d i} f^{i e b}+f^{a e c} f^{c b d}+f^{a e i} f^{i b d} & =0 .
\end{aligned}
$$

The field strength decomposes as

$$
F_{\mu \nu}=F_{\mu \nu}^{A} T^{A}=F_{\mu \nu}^{a} T^{a}+F_{\mu \nu}^{i} T^{i},
$$

with the off-diagonal and diagonal parts given respectively by

$$
\begin{aligned}
& F_{\mu \nu}^{a}=D_{\mu}^{a b} A_{\nu}^{b}-D_{\nu}^{a b} A_{\mu}^{b}+g f^{a b c} A_{\mu}^{b} A_{\nu}^{c}, \\
& F_{\mu \nu}^{i}=\partial_{\mu} A_{\nu}^{i}-\partial_{\nu} A_{\mu}^{i}+g f^{a b i} A_{\mu}^{a} A_{\nu}^{b},
\end{aligned}
$$

where the covariant derivative $D_{\mu}^{a b}$ is defined with respect to the diagonal components $A_{\mu}^{i}$

$$
D_{\mu}^{a b} \equiv \partial_{\mu} \delta^{a b}-g f^{a b i} A_{\mu}^{i}
$$

For the Yang-Mills action one obtains

$$
S_{\mathrm{YM}}=-\frac{1}{4} \int d^{4} x\left(F_{\mu \nu}^{a} F^{\mu \nu a}+F_{\mu \nu}^{i} F^{\mu \nu i}\right) .
$$

In order to write down the interpolating gauge, we shall consider the most general gauge fixing term compatible with the decomposition of the gauge field into diagonal and off-diagonal components, according to eq.(2.1). Let us first establish the nilpotent BRST transformations of the fields. From

$$
\begin{aligned}
s A_{\mu}^{A} & =-D_{\mu}^{A B} c^{B}=-\left(\partial_{\mu} c^{A}+g f^{A B C} A_{\mu}^{B} c^{C}\right) \\
s c^{A} & =\frac{g}{2} f^{A B C} c^{B} c^{C} \\
s \bar{c}^{A} & =b^{A} \\
s b^{A} & =0
\end{aligned}
$$


one easily gets

$$
\begin{array}{rlrl}
s A_{\mu}^{a} & =-\left(D_{\mu}^{a b} c^{b}+g f^{a b c} A_{\mu}^{b} c^{c}+g f^{a b i} A_{\mu}^{b} c^{i}\right), & & s A_{\mu}^{i}=-\left(\partial_{\mu} c^{i}+g f^{i a b} A_{\mu}^{a} c^{b}\right), \\
s c^{a} & =g f^{a b i} c^{b} c^{i}+\frac{g}{2} f^{a b c} c^{b} c^{c}, & & s c^{i}=\frac{g}{2} f^{i a b} c^{a} c^{b}, \\
s \bar{c}^{a}=b^{a}, & & s \bar{c}^{i}=b^{i}, \\
s b^{a}=0, & & s b^{i}=0 .
\end{array}
$$

Here $\left(c^{a}, c^{i}\right)$ are the off-diagonal and the diagonal components of the Faddeev-Popov ghost field, while $\left(\bar{c}^{a}, b^{a}\right)$ and $\left(\bar{c}^{i}, b^{i}\right)$ are the off-diagonal and diagonal antighosts and Lagrange multipliers, respectively. We also observe that the BRST transformations (2.9) have been obtained by their standard form upon projection on the off-diagonal and diagonal components of the fields.

Thus, for the most general BRST invariant gauge fixing term we write

$$
\begin{aligned}
S_{\mathrm{GF}} & =s \int d^{4} x\left[\bar{c}^{a}\left(\partial^{\mu} A_{\mu}^{a}+\frac{\alpha_{1}}{2} b^{a}+\alpha_{2} g f^{a b i} A_{\mu}^{b} A^{i \mu}\right)+\bar{c}^{i}\left(\partial^{\mu} A_{\mu}^{i}+\frac{\alpha_{3}}{2} b^{i}\right)\right. \\
& \left.+\frac{\alpha_{4}}{4} g f^{a b c} c^{a} \bar{c}^{b} \bar{c}^{c}+\frac{\alpha_{5}}{4} g f^{a b i} \bar{c}^{a} \bar{c}^{b} c^{i}+\frac{\alpha_{6}}{2} g f^{a b i} c^{a} \bar{c}^{b} \bar{c}^{i}\right],
\end{aligned}
$$

where $\alpha_{1}, \alpha_{2}, \alpha_{3}, \alpha_{4}, \alpha_{5}, \alpha_{6}$ are six independent parameters. It is not difficult to see that the gauge fixing (2.10) interpolates between several gauges, for instance: the linear covariant gauges, including the Landau gauge as a special case, the maximal Abelian gauge, the modified maximal Abelian gauge and the Curci-Ferrari gauge. This will be checked in the next section, where the various limits for the gauge parameters will be analyzed in detail.

The expression (2.10) is easily worked out and yields

$$
\begin{aligned}
S_{\mathrm{GF}} & =\int d^{4} x\left[b ^ { a } \left(\partial^{\mu} A_{\mu}^{a}+\frac{\alpha_{1}}{2} b^{a}+\alpha_{2} g f^{a b i} A_{\mu}^{b} A^{i \mu}+\frac{\alpha_{4}}{2} g f^{a b c} \bar{c}^{b} c^{c}+\frac{\alpha_{5}}{2} g f^{a b i} \bar{c}^{b} c^{i}\right.\right. \\
& \left.+\frac{\alpha_{6}}{2} g f^{a b i} c^{b} \bar{c}^{i}\right)+b^{i}\left(\partial^{\mu} A_{\mu}^{i}+\frac{\alpha_{3}}{2} b^{i}+\frac{\alpha_{6}}{2} g f^{a b i} c^{a} \bar{c}^{b}\right)+\bar{c}^{a} \partial^{2} c^{a}+\bar{c}^{i} \partial^{2} c^{i} \\
& +g f^{a b c} \partial^{\mu} \bar{c}^{a} c^{b} A_{\mu}^{c}+g f^{a b i} \partial^{\mu} \bar{c}^{a} c^{b} A_{\mu}^{i}-g f^{a b i} \partial^{\mu} \bar{c}^{a} c^{i} A_{\mu}^{b}-g f^{a b i} c^{a} \partial^{\mu} \bar{c}^{i} A_{\mu}^{b} \\
& +\alpha_{2} g f^{a b i} \bar{c}^{a} \partial^{\mu} c^{b} A_{\mu}^{i}+\alpha_{2} g f^{a b i} \bar{c}^{a} \partial^{\mu} c^{i} A_{\mu}^{b}-\alpha_{2} g^{2} f^{a b i} f^{b c j} \bar{c}^{a} c^{c} A_{\mu}^{i} A^{j \mu} \\
& +\alpha_{2} g^{2} f^{a b i} f^{b c d} \bar{c}^{a} c^{d} A_{\mu}^{c} A^{i \mu}+\alpha_{2} g^{2} f^{a b i} f^{b c j} \bar{c}^{a} c^{j} A_{\mu}^{c} A^{i \mu}+\alpha_{2} g^{2} f^{a b i} f^{c d i} \bar{c}^{a} c^{d} A_{\mu}^{c} A^{b \mu} \\
& +\frac{\alpha_{4}}{4} g^{2} f^{a b c} f^{a d i} \bar{c}^{b} \bar{c}^{c} c^{d} c^{i}+\frac{\alpha_{4}}{8} g^{2} f^{a b c} f^{a d e} \bar{c}^{b} \bar{c}^{c} c^{d} c^{e}+\frac{\alpha_{5}}{8} g^{2} f^{a b i} f^{c d i} \bar{c}^{a} \bar{c}^{b} c^{c} c^{d} \\
& \left.+\frac{\alpha_{6}}{2} g^{2} f^{a b i} f^{a c j} \bar{c}^{b} \bar{c}^{i} c^{c} c^{j}+\frac{\alpha_{6}}{4} g^{2} f^{a b i} f^{a c d} \bar{c}^{b} \bar{c}^{i} c^{c} c^{d}\right] .
\end{aligned}
$$

For calculational purposes it is useful to eliminate the Lagrange multipliers. This is done by means of the equations of motion

$$
\begin{aligned}
& \frac{\delta\left(S_{\mathrm{YM}}+S_{\mathrm{GF}}\right)}{\delta b^{a}}=\alpha_{1} b^{a}+\partial^{\mu} A_{\mu}^{a}+\alpha_{2} g f^{a b i} A_{\mu}^{b} A^{i \mu}+\frac{\alpha_{4}}{2} g f^{a b c} \bar{c}^{b} c^{c}+\frac{\alpha_{5}}{2} g f^{a b i} \bar{c}^{b} c^{i}+\frac{\alpha_{6}}{2} g f^{a b i} c^{b} \bar{c}^{i}, \\
& \frac{\delta\left(S_{\mathrm{YM}}+S_{G F}\right)}{\delta b^{i}}=\alpha_{3} b^{i}+\partial^{\mu} A_{\mu}^{i}+\frac{\alpha_{6}}{2} g f^{a b i} c^{a} \bar{c}^{b} .
\end{aligned}
$$


Substituting the expressions (2.12) into the expression (2.11), we find

$$
\begin{aligned}
S_{\mathrm{GF}} & =\int d^{4} x\left[-\frac{1}{2 \alpha_{1}} \partial^{\mu} A_{\mu}^{a} \partial^{\nu} A_{\nu}^{a}-\frac{1}{2 \alpha_{3}} \partial^{\mu} A_{\mu}^{i} \partial^{\nu} A_{\nu}^{i}-\frac{\alpha_{2}}{\alpha_{1}} g f^{a b i} \partial^{\mu} A_{\mu}^{a} A_{\nu}^{b} A^{i \nu}\right. \\
& -\frac{\alpha_{2}^{2}}{2 \alpha_{1}} g^{2} f^{a b i} f^{a c j} A_{\mu}^{b} A^{i \mu} A_{\nu}^{c} A^{j \nu}+\bar{c}^{a} \partial^{2} c^{a}+\bar{c}^{i} \partial^{2} c^{i}+\frac{\alpha_{4}}{2 \alpha_{1}} g f^{a b c} \bar{c}^{a} \partial^{\mu} c^{b} A_{\mu}^{c} \\
& +\left(\frac{\alpha_{4}}{2 \alpha_{1}}+1\right) g f^{a b c} \partial^{\mu} \bar{c}^{a} c^{b} A_{\mu}^{c}+\left(\frac{\alpha_{6}}{2 \alpha_{3}}+1\right) g f^{a b i} \partial^{\mu} \bar{c}^{a} c^{b} A_{\mu}^{i}+\left(\frac{\alpha_{6}}{2 \alpha_{1}}+1\right) g f^{a b i} A_{\mu}^{a} c^{b} \partial^{\mu} \bar{c}^{i} \\
& -\left(\frac{\alpha_{5}}{2 \alpha_{1}}+1\right) g f^{a b i} \partial^{\mu} \bar{c}^{a} c^{i} A_{\mu}^{b}-\frac{\alpha_{6}}{2 \alpha_{1}} g f^{a b i} \partial^{\mu} c^{a} \bar{c}^{i} A_{\mu}^{b}+\left(\frac{\alpha_{6}}{2 \alpha_{3}}+\alpha_{2}\right) g f^{a b i} \bar{c}^{a} \partial^{\mu} c^{b} A_{\mu}^{i} \\
& +\left(\alpha_{2}-\frac{\alpha_{5}}{2 \alpha_{1}}\right) g f^{a b i} \bar{c}^{a} \partial^{\mu} c^{i} A_{\mu}^{b}+\alpha_{2} g^{2}\left(f^{a b i} f^{b c d}+\frac{\alpha_{4}}{2 \alpha_{1}} f^{a b d} f^{b c i}\right) \bar{c}^{a} c^{d} A_{\mu}^{c} A^{i \mu} \\
& +\left(\frac{\alpha_{5}}{2 \alpha_{1}}+1\right) \alpha_{2} g^{2} f^{a b i} f^{b c j} \bar{c}^{a} c^{j} A_{\mu}^{c} A^{i \mu}-\frac{\alpha_{2} \alpha_{6}}{2 \alpha_{1}} g^{2} f^{a b i} f^{a c j} c^{b} \bar{c}^{i} A_{\mu}^{c} A^{j \mu} \\
& -\alpha_{2} g^{2} f^{a b i} f^{b c j} \bar{c}^{a} c^{c} A_{\mu}^{i} A^{j \mu}+\alpha_{2} g^{2} f^{a b i} f^{c d i} \bar{c}^{a} c^{d} A_{\mu}^{c} A^{b \mu}+\frac{\alpha_{4}}{4} g^{2}\left(f^{a b c} f^{a d i}\right. \\
& \left.+\frac{\alpha_{5}}{\alpha_{1}} f^{a b d} f^{a c i}\right) \bar{c}^{b} \bar{c}^{c} c^{d} c^{i}+\frac{g^{2}}{8}\left(\alpha_{5} f^{a b i} f^{c d i}+\alpha_{4} f^{a b e} f^{c d e}+\frac{\alpha_{6}^{2}}{\alpha_{3}} f^{a c i} f^{b d i}\right. \\
& \left.+\frac{\alpha_{4}^{2}}{\alpha_{1}} f^{a c e} f^{b d e}\right) \bar{c}^{a} \bar{c}^{b} c^{c} c^{d}+\frac{\alpha_{6}}{2}\left(1+\frac{\alpha_{5}}{2 \alpha_{1}}\right) g^{2} f^{a b i} f^{a c j} \bar{c}^{b} \bar{c}^{i} c^{c} c^{j}+\frac{\alpha_{6}}{4} g^{2}\left(f^{a b i} f^{a c d}\right. \\
& \left.\left.-\frac{\alpha_{4}}{\alpha_{1}} f^{a b c} f^{a d i}\right) \bar{c}^{b} \bar{c}^{i} c^{c} c^{d}\right] .
\end{aligned}
$$

\section{Interpolating among various gauges.}

Let us now proceed by showing how expression (2.13) can be used to interpolate among various gauges. We begin with the linear covariant gauges, including the Landau gauge.

\subsection{Linear covariant and Landau gauge.}

The linear covariant gauges (LCG) correspond to the following gauge fixing term

$$
S_{\mathrm{LCG}}=s \int d^{4} x \bar{c}^{A}\left(\frac{\alpha}{2} b^{A}+\partial^{\mu} A_{\mu}^{A}\right)
$$

which yields

$$
S_{\mathrm{LCG}}=\int d^{4} x\left[\bar{c}^{A} \partial^{\mu} D_{\mu}^{A B} c^{B}+b^{A}\left(\frac{\alpha}{2} b^{A}+\partial^{\mu} A_{\mu}^{A}\right)\right] .
$$

Eliminating the Lagrange multiplier

$$
S_{\mathrm{LCG}}=\int d^{4} x\left(\bar{c}^{A} \partial^{\mu} D_{\mu}^{A B} c^{B}-\frac{1}{2 \alpha} \partial^{\mu} A_{\mu}^{A} \partial^{\nu} A_{\nu}^{A}\right) .
$$

As usual, the limit $\alpha \rightarrow 0$, defines the Landau gauge. In order to recover the linear covariant gauges from expression (2.13), the values of the gauge parameters should be taken as is shown in Table 1, namely

$$
\alpha_{2}=\alpha_{4}=\alpha_{5}=\alpha_{6}=0,
$$




\begin{tabular}{|c|c|c|c|c|c|c|}
\hline gauge parameter & $\alpha_{1}$ & $\alpha_{2}$ & $\alpha_{3}$ & $\alpha_{4}$ & $\alpha_{5}$ & $\alpha_{6}$ \\
\hline limit & $\alpha$ & 0 & $\alpha$ & $\alpha_{6}$ & $\alpha_{6}$ & 0 \\
\hline
\end{tabular}

Table 1: Values of the gauge parameters for the linear covariant gauges.

and

$$
\alpha_{1}=\alpha_{3}=\alpha
$$

After this, expression (2.13) yields

$$
\begin{aligned}
S_{\mathrm{GF}} & =\int d^{4} x\left(-\frac{1}{2 \alpha} \partial^{\mu} A_{\mu}^{a} \partial^{\nu} A_{\nu}^{a}-\frac{1}{2 \alpha} \partial^{\mu} A_{\mu}^{i} \partial^{\nu} A_{\nu}^{i}+\bar{c}^{a} \partial^{2} c^{a}+\bar{c}^{i} \partial^{2} c^{i}\right. \\
& \left.+g f^{a b c} \partial^{\mu} \bar{c}^{a} c^{b} A_{\mu}^{c}+g f^{a b i} \partial^{\mu} \bar{c}^{a} c^{b} A_{\mu}^{i}+g f^{a b i} A_{\mu}^{a} c^{b} \partial^{\mu} \bar{c}^{i}-g f^{a b i} \partial^{\mu} \bar{c}^{a} c^{i} A_{\mu}^{b}\right) .
\end{aligned}
$$

Now, collecting together both diagonal and off-diagonal indices, we can rewrite the above expression as

$$
S_{\mathrm{GF}}=\int d^{4} x\left(-\frac{1}{2 \alpha} \partial^{\mu} A_{\mu}^{A} \partial^{\nu} A_{\nu}^{A}+\bar{c}^{A} \partial^{2} c^{A}+g f^{A B C} \partial^{\mu} \bar{c}^{A} c^{B} A_{\mu}^{C}\right)
$$

Upon integration by parts of the last term, we obtain

$$
S_{\mathrm{GF}}=\int d^{4} x\left(-\frac{1}{2 \alpha} \partial^{\mu} A_{\mu}^{A} \partial^{\nu} A_{\nu}^{A}+\bar{c}^{A} \partial^{\mu} D_{\mu}^{A B} c^{B}\right)
$$

which is precisely expression (3.3). In particular, the Landau gauge is thus recovered in the limit $\alpha \rightarrow 0$.

\subsection{Curci-Ferrari gauge.}

Let us consider now the Curci-Ferrari gauge. It is a nonlinear covariant gauge, introduced in [6, 7, 8, specified by

$$
S_{\mathrm{CF}}=s \int d^{4} x\left(\bar{c}^{A}\left(\frac{\alpha}{2} b^{A}+\partial^{\mu} A_{\mu}^{A}\right)-\frac{\alpha}{4} g f^{A B C} \bar{c}^{B} c^{C}\right)
$$

namely

$S_{\mathrm{CF}}=\int d^{4} x\left(b^{A}\left(\frac{\alpha}{2} b^{A}+\partial^{\mu} A_{\mu}^{A}-\frac{\alpha}{2} g f^{A B C} \bar{c}^{B} c^{C}\right)+\bar{c}^{A} \partial^{\mu} D_{\mu}^{A B} c^{B}-\frac{\alpha}{8} g^{2} f^{A B C} f^{C D E} \bar{c}^{A} \bar{c}^{B} c^{D} c^{E}\right)$,

which, after elimination of the Lagrange multiplier, becomes

$$
\begin{aligned}
S_{\mathrm{CF}} & =\int d^{4} x\left(-\frac{1}{2 \alpha} \partial^{\mu} A_{\mu}^{A} \partial^{\nu} A_{\nu}^{A}+\bar{c}^{A} \partial^{\mu} D_{\mu}^{A B} c^{B}+\frac{g}{2} g f^{A B C} \partial^{\mu} A_{\mu}^{A} \bar{c}^{B} c^{C}\right. \\
& \left.+\frac{\alpha}{8} g^{2} f^{A E C} f^{C B D} \bar{c}^{A} \bar{c}^{B} c^{D} c^{E}\right)
\end{aligned}
$$

where use has been made of the Jacobi identity (2.2). To obtain the Curci-Ferrari gauge from expression (2.13) the gauge parameters have to be taken as in Table 2, i.e.

$$
\begin{aligned}
& \alpha_{2}=0, \\
& \alpha_{1}=\alpha_{3}=-\alpha_{4}=-\alpha_{5}=-\alpha_{6}=\alpha,
\end{aligned}
$$




\begin{tabular}{|c|c|c|c|c|c|c|}
\hline gauge parameter & $\alpha_{1}$ & $\alpha_{2}$ & $\alpha_{3}$ & $\alpha_{4}$ & $\alpha_{5}$ & $\alpha_{6}$ \\
\hline limit & $-\alpha_{6}$ & 0 & $-\alpha_{6}$ & $\alpha_{6}$ & $\alpha_{6}$ & $-\alpha$ \\
\hline
\end{tabular}

Table 2: Values of the gauge parameters for the Curci-Ferrari gauge.

This leads to

$$
\begin{aligned}
S_{\mathrm{CF}} & =\int d^{4} x\left(-\frac{1}{2 \alpha} \partial^{\mu} A_{\mu}^{a} \partial^{\nu} A_{\nu}^{a}-\frac{1}{2 \alpha} \partial^{\mu} A_{\mu}^{i} \partial^{\nu} A_{\nu}^{i}+\bar{c}^{a} \partial^{2} c^{a}+\bar{c}^{i} \partial^{2} c^{i}-\frac{1}{2} g f^{a b c} \bar{c}^{a} \partial^{\mu} c^{b} A_{\mu}^{c}\right. \\
& +\frac{1}{2} g f^{a b c} \partial^{\mu} \bar{c}^{a} c^{b} A_{\mu}^{c}+\frac{1}{2} g f^{a b i} \partial^{\mu} \bar{c}^{a} c^{b} A_{\mu}^{i}+\frac{1}{2} g f^{a b i} A_{\mu}^{a} c^{b} \partial^{\mu} \bar{c}^{i}-\frac{1}{2} g f^{a b i} \partial^{\mu} \bar{c}^{a} c^{i} A_{\mu}^{b} \\
& +\frac{1}{2} g f^{a b i} \partial^{\mu} c^{a} \bar{c}^{i} A_{\mu}^{b}-\frac{1}{2} g f^{a b i} \bar{c}^{a} \partial^{\mu} c^{b} A_{\mu}^{i}+\frac{1}{2} g f^{a b i} \bar{c}^{a} \partial^{\mu} c^{i} A_{\mu}^{b} \\
& -\frac{\alpha}{4} g^{2}\left(f^{a b c} f^{a d i}-f^{a b d} f^{a c i}\right) \bar{c}^{b} \bar{c}^{c} c^{d} c^{i}-\alpha \frac{g^{2}}{8}\left(f^{a b i} f^{c d i}+f^{a b e} f^{c d e}-f^{a c i} f^{b d i}\right. \\
& \left.\left.-f^{a c e} f^{b d e}\right) \bar{c}^{a} \bar{c}^{b} c^{c} c^{d}-\frac{\alpha}{4} g^{2} f^{a b i} f^{a c j} \bar{c}^{b} \bar{c}^{i} c^{c} c^{j}-\frac{\alpha}{4} g^{2}\left(f^{a b i} f^{a c d}+f^{a b c} f^{a d i}\right) \bar{c}^{b} \bar{c}^{i} c^{c} c^{d}\right)
\end{aligned}
$$

Collecting the various indices and using the Jacobi identities (2.3) for the quartic ghost interaction terms, we obtain

$$
\begin{aligned}
S_{\mathrm{CF}} & =\int d^{4} x\left(-\frac{1}{2 \alpha} \partial^{\mu} A_{\mu}^{A} \partial^{\nu} A_{\nu}^{A}+\bar{c}^{A} \partial^{2} c^{A}-\frac{1}{2} g f^{A B C} \bar{c}^{A} \partial^{\mu} c^{B} A_{\mu}^{C}+\frac{1}{2} g f^{A B C} \partial^{\mu} \bar{c}^{A} c^{B} A_{\mu}^{C}\right. \\
& +\frac{\alpha}{4} g^{2} f^{a b i} f^{a c d} \bar{c}^{b} \bar{c}^{c} c^{d} c^{i}+\alpha \frac{g^{2}}{8}\left(f^{a d e} f^{e b c}+f^{a d i} f^{i b c}\right) \bar{c}^{a} \bar{c}^{b} c^{c} c^{d}-\frac{\alpha}{4} g^{2} f^{a b i} f^{a c j} \bar{c}^{b} \bar{c}^{i} c^{c} c^{j} \\
& \left.-\frac{\alpha}{4} g^{2} f^{a b d} f^{a c i} \bar{c}^{b} \bar{c}^{i} c^{c} c^{d}\right) .
\end{aligned}
$$

In other words

$$
\begin{aligned}
S_{\mathrm{CF}} & =\int d^{4} x\left(-\frac{1}{2 \alpha} \partial^{\mu} A_{\mu}^{A} \partial^{\nu} A_{\nu}^{A}+\bar{c}^{A} \partial^{2} c^{A}-\frac{1}{2} g f^{A B C} \bar{c}^{A} \partial^{\mu} c^{B} A_{\mu}^{C}+\frac{\alpha}{8} g^{2} f^{E A D} f^{E B C} \bar{c}^{A} \bar{c}^{B} c^{C} c^{D}\right. \\
& \left.+\frac{1}{2} g f^{A B C} \partial^{\mu} \bar{c}^{A} c^{B} A_{\mu}^{C}\right) .
\end{aligned}
$$

Finally, integrating the last term of the above expression by parts, eq.([3.15), yields

$$
\begin{aligned}
S_{\mathrm{CF}} & =\int d^{4} x\left(-\frac{1}{2 \alpha} \partial^{\mu} A_{\mu}^{A} \partial^{\nu} A_{\nu}^{A}+\bar{c}^{A} \partial^{\mu} D_{\mu}^{A B} c^{B}+\frac{1}{2} g f^{A B C} \bar{c}^{A} c^{B} \partial^{\mu} A_{\mu}^{C}\right. \\
& \left.+\frac{\alpha}{8} g^{2} f^{E A D} f^{E B C} \bar{c}^{A} \bar{c}^{B} c^{C} c^{D}\right)
\end{aligned}
$$

which reproduces the Curci-Ferrari gauge fixing term, (3.11).

\subsection{Maximal Abelian gauge.}

The so called MAG gauge condition amounts to fixing the value of the covariant derivative, $D_{\mu}^{a b} A^{\mu b}$, of the off-diagonal components by requiring that the functional

$$
\mathcal{R}[A]=(V T)^{-1} \int d^{4} x\left(A_{\mu}^{a} A^{\mu a}\right),
$$


attains a minimum with respect to the local gauge transformations. This corresponds to imposing

$$
D_{\mu}^{a b} A^{\mu b}=0 .
$$

However, this condition being non-linear, a quartic ghost self-interaction term is required for renormalizability purposes. The corresponding gauge fixing term turns out to be [9, 10,

$$
S_{\mathrm{MAG}}^{\mathrm{off}}=s \int d^{4} x\left[\bar{c}^{a}\left(D_{\mu}^{a b} A^{b \mu}+\frac{\alpha}{2} b^{a}\right)-\frac{\alpha}{2} g f^{a b i} \bar{c}^{a} \bar{c}^{b} c^{i}-\frac{\alpha}{4} g f^{a b c} c^{a} \bar{c}^{b} \bar{c}^{c}\right],
$$

and the real MAG, eq.(3.18), is obtained by setting $\alpha=0$ after renormalization. The MAG condition allows for a residual local $U(1)^{N-1}$ invariance with respect to the diagonal subgroup of $S U(N)$. In order to have a complete quantization of the theory, one has to fix this Abelian gauge freedom by means of a suitable further gauge condition on the diagonal components $A_{\mu}^{i}$ of the gauge field. A common choice for the Abelian gauge fixing, also adopted in the lattice papers [11, 12, is the Landau gauge, given by

$$
S_{\mathrm{MAG}}^{\mathrm{diag}}=s \int d^{4} x \bar{c}^{i} \partial_{\mu} A^{i \mu}
$$

The complete gauge fixing is, thus, the sum of expressions (3.19) and (3.20), namely

$$
S_{\mathrm{MAG}}=s \int d^{4} x\left[\bar{c}^{a}\left(D_{\mu}^{a b} A^{b \mu}+\frac{\alpha}{2} b^{a}\right)+\bar{c}^{i} \partial_{\mu} A^{i \mu}-\frac{\alpha}{2} g f^{a b i} \bar{c}^{a} \bar{c}^{b} c^{i}-\frac{\alpha}{4} g f^{a b c} c^{a} \bar{c}^{b} \bar{c}^{c}\right] .
$$

Working out expression (3.21) we find

$$
\begin{aligned}
S_{\mathrm{MAG}} & =\int d^{4} x\left[b^{a}\left(\partial^{\mu} A_{\mu}^{a}-g f^{a b i} A_{\mu}^{b} A^{i \mu}+\frac{\alpha}{2} b^{a}-\alpha g f^{a b i} \bar{c}^{b} c^{i}-\frac{\alpha}{2} g f^{a b c} \bar{c}^{b} c^{c}\right)+b^{i} \partial^{\mu} A_{\mu}^{i}\right. \\
& +\bar{c}^{a} D_{\mu}^{a b} D^{b c \mu} c^{c}+\bar{c}^{i} \partial^{2} c^{i}+g f^{a b i} A_{\mu}^{a} \partial^{\mu} \bar{c}^{b} c^{i}+g f^{a b i} A_{\mu}^{a} \bar{c}^{b} \partial^{\mu} c^{i}+g f^{a b i} A_{\mu}^{a} c^{b} \partial^{\mu} \bar{c}^{i} \\
& +g f^{a b c} \partial^{\mu} \bar{c}^{a} c^{b} A_{\mu}^{c}-g^{2} f^{a b i} f^{b c j} \bar{c}^{a} c^{i} A_{\mu}^{c} A^{j \mu}-g^{2} f^{a b i} f^{b c d} \bar{c}^{a} c^{d} A_{\mu}^{c} A^{i \mu} \\
& -g^{2} f^{a b i} f^{c d i} \bar{c}^{a} c^{d} A_{\mu}^{b} A^{c \mu}-\frac{\alpha}{4} g^{2} f^{a b i} f^{c d i} \bar{c}^{a} \bar{c}^{b} c^{c} c^{d}-\frac{\alpha}{4} g^{2} f^{a b c} f^{a d i} \bar{c}^{b} \bar{c}^{c} c^{d} c^{i} \\
& \left.-\frac{\alpha}{8} g^{2} f^{a b c} f^{a d e} \bar{c}^{b} \bar{c}^{c} c^{d} c^{e}\right] .
\end{aligned}
$$

The task of eliminating the off-diagonal Lagrange multipliers is straightforward. To eliminate the diagonal Lagrange multiplier we have to introduce a parameter $\beta$ via the term $\frac{\beta}{2} b^{i} b^{i}$, and then let $\beta \rightarrow 0$ to recover the transversality of the diagonal gauge field. This will lead to the following gauge fixing

$$
\begin{aligned}
S_{\mathrm{MAG}} & =\lim _{\beta \rightarrow 0} \int d^{4} x\left[-\frac{1}{2 \alpha} \partial^{\mu} A_{\mu}^{a} \partial^{\nu} A_{\nu}^{a}-\frac{1}{2 \beta} \partial^{\mu} A_{\mu}^{i} \partial^{\nu} A_{\nu}^{i}+\frac{g}{\alpha} f^{a b i} \partial^{\mu} A_{\mu}^{a} A_{\nu}^{a} A^{i \nu}\right. \\
& -\frac{g^{2}}{2 \alpha} f^{a b i} f^{a c j} A_{\mu}^{b} A^{i \mu} A_{\nu}^{c} A^{j \nu}+\bar{c}^{a} D_{\mu}^{a b} D^{b c \mu} c^{c}+\bar{c}^{i} \partial^{2} c^{i}+g f^{a b i} A_{\mu}^{a} c^{b} \partial^{\mu} \bar{c}^{i}+\frac{1}{2} g f^{a b c} \partial^{\mu} \bar{c}^{a} c^{b} A_{\mu}^{c} \\
& -\frac{1}{2} g f^{a b c} \bar{c}^{a} \partial^{\mu} c^{b} A_{\mu}^{c}-\frac{g^{2}}{2}\left(2 f^{a b i} f^{b c d}-f^{a b d} f^{b c i}\right) \bar{c}^{a} c^{d} A_{\mu}^{c} A^{i \mu}-g^{2} f^{a b i} f^{c d i} \bar{c}^{a} c^{d} A_{\mu}^{b} A^{c \mu} \\
& -\frac{\alpha}{4} g^{2}\left(2 f^{a b i} f^{a c d}+f^{a b c} f^{a d i}\right) \bar{c}^{b} \bar{c}^{c} c^{d} c^{i} \\
& \left.-\frac{\alpha}{8} g^{2}\left(2 f^{b c i} f^{d e i}+f^{a b c} f^{a d e}-f^{a b d} f^{a c e}\right) \bar{c}^{b} \bar{c}^{c} c^{d} c^{e}\right] .
\end{aligned}
$$




\begin{tabular}{|c|c|c|c|c|c|c|}
\hline gauge parameter & $\alpha_{1}$ & $\alpha_{2}$ & $\alpha_{3}$ & $\alpha_{4}$ & $\alpha_{5}$ & $\alpha_{6}$ \\
\hline limit & $\alpha$ & -1 & $\beta \stackrel{\text { end }}{\longrightarrow} 0$ & $-\alpha$ & $-2 \alpha$ & 0 \\
\hline
\end{tabular}

Table 3: Values of the gauge parameters for the maximal Abelian gauge.

Expression (3.23) is recovered from the interpolating gauge fixing (2.13), once the gauge parameters are taken as in Table 3 ,

$$
\begin{aligned}
& \alpha_{1}=-\alpha_{4}=-\frac{\alpha_{5}}{2}=\alpha, \\
& \alpha_{2}=-1, \alpha_{6}=0,
\end{aligned}
$$

and then

$$
\alpha_{3}=\beta \rightarrow 0
$$

Therefore, expression (2.13) becomes

$$
\begin{aligned}
S_{\mathrm{GF}} & =\lim _{\beta \rightarrow 0} \int d^{4} x\left[-\frac{1}{2 \alpha} \partial^{\mu} A_{\mu}^{a} \partial^{\nu} A_{\nu}^{a}-\frac{1}{2 \beta} \partial^{\mu} A_{\mu}^{i} \partial^{\nu} A_{\nu}^{i}+\frac{1}{\alpha} g f^{a b i} \partial^{\mu} A_{\mu}^{a} A_{\nu}^{b} A^{i \nu}\right. \\
& -\frac{1}{2 \alpha} g^{2} f^{a b i} f^{a c j} A_{\mu}^{b} A^{i \mu} A_{\nu}^{c} A^{j \nu}+\bar{c}^{a} \partial^{2} c^{a}+\bar{c}^{i} \partial^{2} c^{i}-\frac{1}{2} g f^{a b c} \bar{c}^{a} \partial^{\mu} c^{b} A_{\mu}^{c} \\
& +\frac{1}{2} g f^{a b c} \partial^{\mu} \bar{c}^{a} c^{b} A_{\mu}^{c}+g f^{a b i} \partial^{\mu} \bar{c}^{a} c^{b} A_{\mu}^{i}+g f^{a b i} A_{\mu}^{a} c^{b} \partial^{\mu} \bar{c}^{i}-g f^{a b i} \bar{c}^{a} \partial^{\mu} c^{b} A_{\mu}^{i} \\
& -g^{2}\left(f^{a b i} f^{b c d}-\frac{1}{2} f^{a b d} f^{b c i}\right) \bar{c}^{a} c^{d} A_{\mu}^{c} A^{i \mu}+g^{2} f^{a b i} f^{b c j} \bar{c}^{a} c^{c} A_{\mu}^{i} A^{j \mu}-g^{2} f^{a b i} f^{c d i} \bar{c}^{a} c^{d} A_{\mu}^{c} A^{b \mu} \\
& -\frac{\alpha}{4} g^{2}\left(f^{a b c} f^{a d i}-2 f^{a b d} f^{a c i}\right) \bar{c}^{b} \bar{c}^{c} c^{d} c^{i} \\
& \left.-\alpha \frac{g^{2}}{8}\left(2 f^{a b i} f^{c d i}+f^{a b e} f^{c d e}-f^{a c e} f^{b d e}\right) \bar{c}^{a} \bar{c}^{b} c^{c} c^{d}\right]
\end{aligned}
$$

which, after rearranging some indices, yields the expression (3.23).

\subsection{Modified maximal Abelian gauge.}

For completeness, let us include in the present analysis also a slightly modified version of the maximal Abelian gauge. This gauge, discussed in [13], is obtained by making the following choice for the diagonal gauge fixing term

$$
S_{\mathrm{MAG}}^{\mathrm{diag}}=s \int d^{4} x\left(\bar{c}^{i} \partial^{\mu} A_{\mu}^{i}+g f^{a b i} \bar{c}^{a} A_{\mu}^{b} A^{i \mu}\right),
$$

Thus, for the complete gauge gauge fixing in the modified maximal Abelian gauge, MMAG, we have

$$
S_{\mathrm{MMAG}}=s \int d^{4} x\left(\bar{c}^{a}\left(\partial^{\mu} A_{\mu}^{a}+\frac{\alpha}{2} b^{a}\right)+\bar{c}^{i} \partial_{\mu} A^{i \mu}-\frac{\alpha}{2} g f^{a b i} \bar{c}^{a} \bar{c}^{b} c^{i}-\frac{\alpha}{4} g f^{a b c} c^{a} \bar{c}^{b} \bar{c}^{c}\right),
$$

namely

$$
S_{\mathrm{MMAG}}=\int d^{4} x\left[b^{a}\left(\partial^{\mu} A_{\mu}^{a}+\frac{\alpha}{2} b^{a}-\alpha g f^{a b i} \bar{c}^{b} c^{i}-\frac{\alpha}{2} g f^{a b c} \bar{c}^{b} c^{c}\right)+b^{i} \partial_{\mu} A^{i \mu}+\bar{c}^{a} \partial^{\mu} D_{\mu}^{a b} c^{b}\right.
$$




\begin{tabular}{|c|c|c|c|c|c|c|}
\hline gauge parameter & $\alpha_{1}$ & $\alpha_{2}$ & $\alpha_{3}$ & $\alpha_{4}$ & $\alpha_{5}$ & $\alpha_{6}$ \\
\hline limit & $\alpha$ & 0 & $\beta \stackrel{\text { end }}{\rightarrow} 0$ & $-\alpha$ & $-2 \alpha$ & 0 \\
\hline
\end{tabular}

Table 4: Values of the gauge parameters for the modified maximal Abelian gauge.

$$
\begin{aligned}
& +\bar{c}^{i} \partial^{2} c^{i}+g f^{a b i} A_{\mu}^{a} c^{b} \partial^{\mu} \bar{c}^{i}-g f^{a b c} \partial^{\mu} \bar{c}^{a} A_{\mu}^{b} c^{c}-g f^{a b i} \partial^{\mu} \bar{c}^{a} A_{\mu}^{b} c^{i}-\frac{\alpha}{4} g^{2} f^{a b c} f^{a d i} \bar{c}^{b} \bar{c}^{c} c^{d} c^{i} \\
& \left.-\frac{\alpha}{4} g^{2} f^{a b i} f^{c d i} \bar{c}^{a} \bar{c}^{b} c^{c} c^{d}-\frac{\alpha}{8} g^{2} f^{a b e} f^{c d e} \bar{c}^{a} \bar{c}^{b} c^{c} c^{d}\right]
\end{aligned}
$$

which, upon elimination of the Lagrange multipliers, becomes

$$
\begin{aligned}
S_{\mathrm{MMAG}} & =\lim _{\beta \rightarrow 0} \int d^{4} x\left[-\frac{1}{2 \alpha} \partial^{\mu} A_{\mu}^{a} \partial^{\nu} A_{\nu}^{a}-\frac{1}{2 \beta} \partial_{\mu} A^{i \mu} \partial_{\nu} A^{i \nu}+\bar{c}^{a} \partial^{\mu} D_{\mu}^{a b} c^{b}+\bar{c}^{i} \partial^{2} c^{i}\right. \\
& +g f^{a b i} A_{\mu}^{a} c^{b} \partial^{\mu} \bar{c}^{i}-\frac{g}{2} f^{a b c} \bar{c}^{a} \partial^{\mu} c^{b} A_{\mu}^{c}+\frac{g}{2} f^{a b c} \partial^{\mu} \bar{c}^{a} c^{b} A_{\mu}^{c}+g f^{a b i} \bar{c}^{a} A_{\mu}^{b} \partial^{\mu} c^{i} \\
& -\frac{\alpha}{4} g^{2}\left(2 f^{a b i} f^{a c d}+f^{a b c} f^{a d i}\right) \bar{c}^{b} \bar{c}^{c} c^{d} c^{i} \\
& \left.-\frac{\alpha}{8} g^{2}\left(2 f^{a b i} f^{c d i}+f^{a b e} f^{c d e}+f^{a d e} f^{b c e}\right) \bar{c}^{a} \bar{c}^{b} c^{c} c^{d}\right] .
\end{aligned}
$$

Again, expression (3.30) follows from (2.13) by setting the gauge parameters as in Table 4,

$$
\begin{aligned}
& \alpha_{1}=-\alpha_{4}=-\frac{\alpha_{5}}{2}=\alpha, \\
& \alpha_{2}=\alpha_{6}=0,
\end{aligned}
$$

and

$$
\alpha_{3}=\beta \rightarrow 0
$$

Thus

$$
\begin{aligned}
S_{\mathrm{GF}} & =\lim _{\beta \rightarrow 0} \int d^{4} x\left(-\frac{1}{2 \alpha} \partial^{\mu} A_{\mu}^{a} \partial^{\nu} A_{\nu}^{a}-\frac{1}{2 \beta} \partial^{\mu} A_{\mu}^{i} \partial^{\nu} A_{\nu}^{i}+\bar{c}^{a} \partial^{2} c^{a}+\bar{c}^{i} \partial^{2} c^{i}-\frac{1}{2} g f^{a b c} \bar{c}^{a} \partial^{\mu} c^{b} A_{\mu}^{c}\right. \\
& +\frac{1}{2} g f^{a b c} \partial^{\mu} \bar{c}^{a} c^{b} A_{\mu}^{c}+g f^{a b i} \partial^{\mu} \bar{c}^{a} c^{b} A_{\mu}^{i}+g f^{a b i} A_{\mu}^{a} c^{b} \partial^{\mu} \bar{c}^{i}+g f^{a b i} \bar{c}^{a} \partial^{\mu} c^{i} A_{\mu}^{b} \\
& -\frac{\alpha}{4} g^{2}\left(f^{a b c} f^{a d i}-2 f^{a b d} f^{a c i}\right) \bar{c}^{b} \bar{c}^{c} c^{d} c^{i} \\
& \left.-\alpha \frac{g^{2}}{8}\left(2 f^{a b i} f^{c d i}+f^{a b e} f^{c d e}-f^{a c e} f^{b d e}\right) \bar{c}^{a} \bar{c}^{b} c^{c} c^{d}\right) .
\end{aligned}
$$

By rearranging the indices of the quartic ghost terms by means of the Jacobi identities (2.3), it is then easy to show that

$$
S_{\mathrm{GF}}=S_{\mathrm{MMAG}} .
$$

\section{Multiplicative renormalizability of the interpolating gauge.}

To prove renormalizability, let us first write down the Slavnov-Taylor identity corresponding to the BRST invariance of the classical action. Following the algebraic renormalization setup [14, 
we introduce a set of external sources coupled to the nonlinear BRST transformations (2.9) of the fields,

$$
S_{\mathrm{ext}}=s \int d^{4} x\left(-\Omega^{a \mu} A_{\mu}^{a}-\Omega^{i \mu} A_{\mu}^{i}+L^{a} c^{a}+L^{i} c^{i}\right)
$$

with

$$
s \Omega_{\mu}^{a}=s \Omega_{\mu}^{i}=s L^{a}=s L^{i}=0
$$

or

$$
\begin{aligned}
S_{\mathrm{ext}} & =\int d^{4} x\left[-\Omega^{a \mu}\left(D_{\mu}^{a b} c^{b}+g f^{a b c} A_{\mu}^{b} c^{c}+g f^{a b i} A_{\mu}^{b} c^{i}\right)-\Omega^{i \mu}\left(\partial_{\mu} c^{i}+g f^{a b i} A_{\mu}^{a} c^{b}\right)\right. \\
& \left.+L^{a}\left(g f^{a b i} c^{b} c^{i}+\frac{g}{2} f^{a b c} c^{b} c^{c}\right)+\frac{g}{2} f^{a b i} c^{a} c^{b} L^{i}\right] .
\end{aligned}
$$

Therefore, it follows that the complete action $\Sigma$

$$
\Sigma=S_{Y M}+S_{G F}+S_{e x t},
$$

obeys the Slavnov-Taylor identity

$$
\mathcal{S}(\Sigma)=\int d^{4} x\left(\frac{\delta \Sigma}{\delta \Omega^{a \mu}} \frac{\delta \Sigma}{\delta A_{\mu}^{a}}+\frac{\delta \Sigma}{\delta \Omega^{i \mu}} \frac{\delta \Sigma}{\delta A_{\mu}^{i}}+\frac{\delta \Sigma}{\delta L^{a}} \frac{\delta \Sigma}{\delta c^{a}}+\frac{\delta \Sigma}{\delta L^{i}} \frac{\delta \Sigma}{\delta c^{i}}+b^{a} \frac{\delta \Sigma}{\delta \bar{c}^{a}}+b^{i} \frac{\delta \Sigma}{\delta \bar{c}^{i}}\right)=0 .
$$

In order to characterize the most general invariant counterterm which can be freely added to any order of perturbation theory, we perturb the classical action $\Sigma$ by adding an arbitrary integrated local polynomial $\Sigma^{\text {count }}$ in the fields and external sources of dimension bounded by four and with zero ghost number, and we require that the perturbed action $\left(\Sigma+\eta \Sigma^{\text {count }}\right)$ satisfies the same Ward identities as $\Sigma$ to the first order in the perturbation parameter $\eta$, being,

$$
\mathcal{S}\left(\Sigma+\eta \Sigma^{\mathrm{count}}\right)=0+O\left(\eta^{2}\right)
$$

This amounts to imposing the following conditions on $\Sigma^{\text {count }}$

$$
\mathcal{S}_{\Sigma} \Sigma^{\text {count }}=0
$$

where $\mathcal{S}_{\Sigma}$ is the linearized Slavnov-Taylor nilpotent operator,

$$
\begin{gathered}
\mathcal{S}_{\Sigma}=\int d^{4} x\left(\frac{\delta \Sigma}{\delta \Omega^{\mu a}} \frac{\delta}{\delta A_{\mu}^{a}}+\frac{\delta \Sigma}{\delta A_{\mu}^{a}} \frac{\delta}{\delta \Omega^{\mu a}}+\frac{\delta \Sigma}{\delta \Omega^{\mu i}} \frac{\delta}{\delta A_{\mu}^{i}}+\frac{\delta \Sigma}{\delta A_{\mu}^{i}} \frac{\delta}{\delta \Omega^{\mu i}}+\frac{\delta \Sigma}{\delta L^{a}} \frac{\delta}{\delta c^{a}}\right. \\
\left.+\frac{\delta \Sigma}{\delta c^{a}} \frac{\delta}{\delta L^{a}}+\frac{\delta \Sigma}{\delta L^{i}} \frac{\delta}{\delta c^{i}}+\frac{\delta \Sigma}{\delta c^{i}} \frac{\delta}{\delta L^{i}}+b^{a} \frac{\delta}{\delta \bar{c}^{a}}+b^{i} \frac{\delta}{\delta \bar{c}^{i}}\right), \\
\mathcal{S}_{\Sigma} \mathcal{S}_{\Sigma}=0 .
\end{gathered}
$$

From the conditions (4.7), it turns out that the most general invariant counterterm $\Sigma^{\text {count }}$ can be written as

$$
\Sigma^{\text {count }}=a_{0} S_{\mathrm{YM}}+\mathcal{S}_{\Sigma} \Delta^{-1}
$$




\begin{tabular}{|c|c|c|c|c|c|c|}
\hline & $A$ & $c$ & $\bar{c}$ & $b$ & $\Omega$ & $L$ \\
\hline dimension & 1 & 0 & 2 & 2 & 3 & 4 \\
ghost number & 0 & 1 & -1 & 0 & -1 & -2 \\
\hline
\end{tabular}

Table 5: Dimensions and ghost numbers

where $\Delta^{-1}$ is a local polynomial in the fields and sources, with dimension four and ghost number, which reads

$$
\begin{aligned}
\Delta^{-1} & =\int d^{4} x\left(a_{1} \frac{\alpha_{1}}{2} b^{a} \bar{c}^{a}+a_{2} \alpha_{2} g f^{a b i} \bar{c}^{a} A_{\mu}^{b} A^{i \mu}+a_{3} \frac{\alpha_{3}}{2} b^{i} \bar{c}^{i}+a_{4} \frac{\alpha_{4}}{4} g f^{a b c} \bar{c}^{a} \bar{c}^{b} c^{c}\right. \\
& +a_{5} \frac{\alpha_{5}}{4} g f^{a b i} \bar{c}^{a} \bar{c}^{b} c^{i}+a_{6} \frac{\alpha_{6}}{2} g f^{a b i} c^{a} \bar{c}^{b} \bar{c}^{i}+a_{7} \partial^{\mu} A_{\mu}^{a} \bar{c}^{a}+a_{8} \partial^{\mu} A_{\mu}^{i} \bar{c}^{i}+a_{9} \Omega_{\mu}^{a} A^{a \mu}+a_{10} \Omega_{\mu}^{i} A^{i \mu} \\
& \left.+a_{11} L^{a} c^{a}+a_{12} L^{i} c^{i}\right)
\end{aligned}
$$

where $a_{i} \mid i \in\{0,1, \ldots, 12\}$ are independent parameters. These parameters can be reabsorbed by means of a multiplicative renormalization of the gauge coupling constant, of the gauge parameters, and of the fields and sources, according to

$$
\Sigma\left(\Phi_{0}, \phi_{0}, J_{0}, j_{0}, \xi_{0}\right)=\Sigma(\Phi, \phi, J, j, \xi)+\eta \Sigma^{\operatorname{count}}(\Phi, \phi, J, j, \xi)
$$

where

$$
\begin{aligned}
\Phi_{0} & =Z_{\Phi}^{1 / 2} \Phi, \\
\phi_{0} & =\tilde{Z}_{\phi}^{1 / 2} \phi, \\
J_{0} & =Z_{J} J, \\
j_{0} & =\tilde{Z}_{j} j, \\
\xi_{0} & =Z_{\xi} \xi,
\end{aligned}
$$

with

$$
\begin{aligned}
\Phi & =\left\{A^{a}, b^{a}, c^{a}, \bar{c}^{a}\right\}, \\
\phi & =\left\{A^{i}, b^{i}, c^{i}, \bar{c}^{i}\right\}, \\
J & =\left\{\Omega^{a}, L^{a}\right\}, \\
j & =\left\{\Omega^{i}, L^{i}\right\}, \\
\xi & =\left\{g, \alpha_{1}, \alpha_{2}, \alpha_{3}, \alpha_{4}, \alpha_{5}, \alpha_{6}\right\} .
\end{aligned}
$$

For the renormalization of the off-diagonal and diagonal gluons, and of the coupling constant $g$, we find, respectively,

$$
\begin{aligned}
Z_{A}^{1 / 2} & =1+\eta\left(\frac{a_{0}}{2}+a_{9}\right) \\
\tilde{Z}_{A}^{1 / 2} & =1+\eta\left(\frac{a_{0}}{2}+a_{10}\right), \\
Z_{g} & =1-\eta \frac{a_{0}}{2}
\end{aligned}
$$


while the Lagrange multipliers renormalize as follows

$$
\begin{aligned}
& Z_{b}^{1 / 2}=1+\eta\left(a_{7}-\frac{a_{0}}{2}\right), \\
& \tilde{Z}_{b}^{1 / 2}=1+\eta\left(a_{8}-\frac{a_{0}}{2}\right) .
\end{aligned}
$$

The renormalization factors of the gauge parameters are

$$
\begin{aligned}
& Z_{\alpha_{1}}=1+\eta\left(a_{0}+a_{1}-2 a_{7}\right), \\
& Z_{\alpha_{2}}=1+\eta\left(a_{2}-a_{7}\right), \\
& Z_{\alpha_{3}}=1+\eta\left(a_{0}+a_{3}-2 a_{8}\right), \\
& Z_{\alpha_{4}}=1+\eta\left(a_{0}+a_{4}-2 a_{7}\right), \\
& Z_{\alpha_{5}}=1+\eta\left(a_{0}+a_{5}-2 a_{7}\right), \\
& Z_{\alpha_{6}}=1+\eta\left(a_{0}+a_{6}-a_{7}-a_{8}\right) .
\end{aligned}
$$

The renormalization of the ghost and anti-ghost fields are constrained by the following selfconsistent relations

$$
\begin{aligned}
& Z_{\bar{c}}^{1 / 2} Z_{c}^{1 / 2}=1+\eta\left(a_{7}-a_{11}\right), \\
& \tilde{Z}_{\bar{c}}^{1 / 2} \tilde{Z}_{c}^{1 / 2}=1+\eta\left(a_{8}-a_{12}\right), \\
& Z_{\bar{c}}^{1 / 2} \tilde{Z}_{c}^{1 / 2}=1+\eta\left(a_{7}-a_{12}\right), \\
& \tilde{Z}_{\bar{c}}^{1 / 2} Z_{c}^{1 / 2}=1+\eta\left(a_{8}-a_{11}\right),
\end{aligned}
$$

which give

$$
\begin{aligned}
& Z_{c}^{1 / 2}=1+\eta\left(\frac{a_{7}}{2}+\frac{a_{8}}{2}-\frac{a_{11}}{2}+\frac{a_{12}}{2}\right), \\
& \tilde{Z}_{c}^{1 / 2}=1+\eta\left(\frac{a_{7}}{2}+\frac{a_{8}}{2}+\frac{a_{11}}{2}-\frac{a_{12}}{2}\right), \\
& Z_{\bar{c}}^{1 / 2}=1+\eta\left(\frac{a_{7}}{2}-\frac{a_{8}}{2}-\frac{a_{11}}{2}-\frac{a_{12}}{2}\right), \\
& \tilde{Z}_{\bar{c}}^{1 / 2}=1+\eta\left(-\frac{a_{7}}{2}+\frac{a_{8}}{2}-\frac{a_{11}}{2}-\frac{a_{12}}{2}\right) .
\end{aligned}
$$

Finally, for the renormalization of the external sources

$$
\begin{aligned}
& Z_{\Omega}=1-\eta\left(\frac{a_{7}}{2}+\frac{a_{8}}{2}+a_{9}+\frac{a_{11}}{2}+\frac{a_{12}}{2}\right), \\
& \tilde{Z}_{\Omega}=1-\eta\left(\frac{a_{7}}{2}+\frac{a_{8}}{2}+a_{10}+\frac{a_{11}}{2}+\frac{a_{12}}{2}\right), \\
& Z_{L}=1+\eta\left(\frac{a_{0}}{2}-a_{7}-a_{8}-a_{12}\right), \\
& \tilde{Z}_{L}=1+\eta\left(\frac{a_{0}}{2}-a_{7}-a_{8}-a_{11}\right) .
\end{aligned}
$$

This completes the proof of the multiplicative renormalization of the interpolating gauge fixing. 


\section{One loop renormalization.}

We now record the details of the explicit renormalization of the interpolating gauge at one loop which requires the computation of the renormalization constants $Z_{A}, \tilde{Z}_{A}, Z_{\psi}, Z_{g}, Z_{c}, Z_{\bar{c}}, \tilde{Z}_{c}, \tilde{Z}_{\bar{c}}$ and $Z_{\alpha_{i}}$, with $i=1, \ldots, 6$, and where $Z_{\psi}$ is the quark wave function renormalization constant ${ }^{\dagger}$. Given the explicit form of the Lagrangian we do not need to consider the renormalization of all three- and four-point interactions. The procedure we adopt is to determine the renormalization constants of the fields and of the gauge parameters $\alpha_{1}, \alpha_{3}$ before deducing the explicit values of the remaining four parameters $\alpha_{2}, \alpha_{4}, \alpha_{5}, \alpha_{6}$. We use dimensional regularization in $d=4-2 \epsilon$ and subtract the divergences using the $\overline{\mathrm{MS}}$ scheme. In previous work we carried out similar renormalization in the Curci-Ferrari gauge and MAG, 15, 16, 4, 17, using the Mincer algorithm, 18, 19, written in FORM, 20, where the diagrams for the appropriate Green's function were generated by the QGRAF package, 21]. However, to determine the full interpolating gauge renormalization constants it transpired that we needed to renormalize several four-point interactions in addition to various three-point functions. In this instance the MINCER algorithm is not fully appropriate since it can only be applied to two-point functions with massless propagators. For a four-point function one would therefore need to nullify two external momenta which could potentially introduce spurious infrared divergences. To circumvent this difficulty for the four-point function renormalization, we applied an alternative algorithm, still in dimensional regularization, which involved nullifying all external momenta but systematically introducing a temporary infrared mass regularization according to the approach of [22, 23. This involved repeatedly replacing massless propagators by massive ones via

$$
\frac{1}{(k-p)^{2}}=\frac{1}{\left(k^{2}-m^{2}\right)}+\frac{\left(2 k p-p^{2}-m^{2}\right)}{(k-p)^{2}\left(k^{2}-m^{2}\right)}
$$

until the contributions from the last term are finite and can be neglected by Weinberg's theorem, 24. The resulting one loop vacuum bubble Feynman diagrams are then elementary to evaluate. For the three-point functions we will still nullify an external momentum but we will do so only in Green's functions where we know no infrared singularities will be introduced. In either approach we will still use the method of [25] to extract the explicit renormalization constants. This involves computing the Green's functions in terms of bare quantities before transforming to renormalized variables and parameters. The form of the tree term then allows one to fix the renormalization constants associated with that Green's function. This approach avoids the need to consider subtractions and moreover is more appropriate to performing automatic symbolic manipulation computations. One additional point arising from using the method of [25] is that we cannot renormalize $\alpha_{5}$ itself since it never appears as an isolated coefficient in one type of interaction. However, since it appears in the combination $\left(1+\alpha_{5} /\left(2 \alpha_{1}\right)\right)$ we have chosen to define a new parameter

$$
\bar{\alpha}_{5}=1+\frac{\alpha_{5}}{2 \alpha_{1}}
$$

and determined its renormalization constant $Z_{\bar{\alpha}_{5}}$. Clearly this choice does not invalidate the renormalizability of the interpolating gauge. We note that to recover the various gauges discussed in Sect. 2 the value of $\bar{\alpha}_{5}$ becomes $1, \frac{1}{2}, 0$ and 0 respectively in the linear covariant, Curci-Ferrari, maximal Abelian and modified maximal Abelian gauges.

\footnotetext{
${ }^{\dagger}$ Although we did not consider matter fields in the previous sections, it can be easily checked that the interpolating gauge fixing term (2.13) remains renormalizable if spinor fields are included.
} 
Since we are considering a gauge where the color group is split into diagonal and off-diagonal sectors we briefly recall the essential properties of the group algebra which is discussed in more depth in [17. In addition to the usual color group Casimirs $C_{F}, C_{A}$ and $T_{F}$ for a general color group we define the dimensions of the centre of the group as $N_{A}^{d}$ and the dimension of the off-diagonal sector as $N_{A}^{o}$. Clearly

$$
N_{A}^{d}+N_{A}^{o}=N_{A}
$$

where $N_{A}$ is the dimension of the adjoint representation. For instance, for $S U(N)$ one has $N_{A}^{d}=N-1$ and $N_{A}^{o}=N(N-1)$. Consequently, [17, one can show that

$$
\begin{aligned}
f^{i a b} f^{i a b} & =N_{A}^{d} C_{A}, \quad f^{a b c} f^{a b c}=\left[N_{A}^{o}-2 N_{A}^{d}\right] C_{A}, f^{i c d} f^{j c d}=C_{A} \delta^{i j}, \\
f^{a c j} f^{b c j} & =\frac{N_{A}^{d}}{N_{A}^{o}} C_{A} \delta^{a b}, f^{a c d} f^{b c d}=\frac{\left[N_{A}^{o}-2 N_{A}^{d}\right]}{N_{A}^{o}} C_{A} \delta^{a b}, \\
f^{a p q} f^{b p r} f^{c q r} & =\frac{\left[N_{A}^{o}-3 N_{A}^{d}\right]}{2 N_{A}^{o}} C_{A} f^{a b c}, f^{a p q} f^{b p i} f^{c q i}=\frac{N_{A}^{d}}{2 N_{A}^{o}} C_{A} f^{a b c}, \\
f^{i p q} f^{b p r} f^{c q r} & =\frac{\left[N_{A}^{o}-2 N_{A}^{d}\right]}{2 N_{A}^{o}} C_{A} f^{i b c}, f^{i p q} f^{b p j} f^{c q j}=\frac{N_{A}^{d}}{N_{A}^{o}} C_{A} f^{i b c}, \\
\operatorname{Tr}\left(T^{a} T^{b}\right) & =T_{F} \delta^{a b}, \operatorname{Tr}\left(T^{a} T^{i}\right)=0, \operatorname{Tr}\left(T^{i} T^{j}\right)=T_{F} \delta^{i j}, \\
T^{i} T^{i} & =\frac{T_{F}}{N_{F}} N_{A}^{d} I, T^{a} T^{a}=\left[C_{F}-\frac{T_{F}}{N_{F}} N_{A}^{d}\right] I, \\
T^{b} T^{a} T^{b} & =\left[C_{F}-\frac{C_{A}}{2}-\frac{T_{F}}{N_{F}} N_{A}^{d}+\frac{C_{A} N_{A}^{d}}{2 N_{A}^{o}}\right] T^{a}, \\
T^{i} T^{a} T^{i} & =\left[\frac{T_{F}}{N_{F}} N_{A}^{d}-\frac{C_{A} N_{A}^{d}}{2 N_{A}^{o}}\right] T^{a}, \\
T^{a} T^{i} T^{a} & =\left[\frac{T_{F}}{N_{F}} N_{A}^{o}-\frac{C_{A}}{2}\right] T^{i}, T^{j} T^{i} T^{j}=\frac{T_{F}}{N_{F}} N_{A}^{d} T^{i},
\end{aligned}
$$

where $N_{F}$ is the dimension of the fundamental representation and satisfies

$$
C_{F} N_{F}=\left[N_{A}^{o}+N_{A}^{d}\right] T_{F} .
$$

For completeness we note that we have assumed that $f^{a c d} f^{b c d}$ is proportional to $\delta^{a b}$ which is certainly true in $S U(2)$ and we have checked that it is valid in $S U(3)$, 17. Moreover, we have assumed that $T^{i} T^{i}$ is proportional to the unit matrix, $I$, which is certainly true for $S U(N)$.

Before turning to the explicit discussion of how we renormalized the interpolating gauge Lagrangian we define the anomalous dimensions of the wave functions and parameters in terms of the renormalization constants we will compute. We have

$$
\begin{gathered}
\gamma_{\phi}(a)=\left[\beta(a) \frac{\partial}{\partial a}+\sum_{n} \alpha_{n} \gamma_{\alpha_{n}}(a) \frac{\partial}{\partial \alpha_{n}}\right] \ln Z_{\phi}, \\
\gamma_{\alpha_{1}}(a)=\left[1-\alpha_{1} \frac{\partial}{\partial \alpha_{1}} \ln Z_{\alpha_{1}}\right]^{-1}\left[-\gamma_{A}(a)+\beta(a) \frac{\partial}{\partial a}+\sum_{n^{\prime}} \alpha_{n} \gamma_{\alpha_{n}}(a) \frac{\partial}{\partial \alpha_{n}}\right] \ln Z_{\alpha_{1}},
\end{gathered}
$$




$$
\begin{aligned}
\gamma_{\alpha_{2}}(a) & =-\left[1+\alpha_{2} \frac{\partial}{\partial \alpha_{2}} \ln Z_{\alpha_{2}}\right]^{-1}\left[\beta(a) \frac{\partial}{\partial a}+\sum_{n^{\prime}} \alpha_{n} \gamma_{\alpha_{n}}(a) \frac{\partial}{\partial \alpha_{n}}\right] \ln Z_{\alpha_{2}} \\
\gamma_{\alpha_{3}}(a) & =\left[1-\alpha_{3} \frac{\partial}{\partial \alpha_{3}} \ln Z_{\alpha_{3}}\right]^{-1}\left[-\gamma_{A}(a)+\beta(a) \frac{\partial}{\partial a}+\sum_{n^{\prime}} \alpha_{n} \gamma_{\alpha_{n}}(a) \frac{\partial}{\partial \alpha_{n}}\right] \ln Z_{\alpha_{3}} \\
\gamma_{\alpha_{4}}(a) & =-\left[1+\alpha_{4} \frac{\partial}{\partial \alpha_{4}} \ln Z_{\alpha_{4}}\right]^{-1}\left[\beta(a) \frac{\partial}{\partial a}+\sum_{n^{\prime}} \alpha_{n} \gamma_{\alpha_{n}}(a) \frac{\partial}{\partial \alpha_{n}}\right] \ln Z_{\alpha_{4}} \\
\gamma_{\bar{\alpha}_{5}}(a) & =-\left[1+\bar{\alpha}_{5} \frac{\partial}{\partial \bar{\alpha}_{5}} \ln Z_{\bar{\alpha}_{5}}\right]^{-1}\left[\beta(a) \frac{\partial}{\partial a}+\sum_{n^{\prime}} \alpha_{n} \gamma_{\alpha_{n}}(a) \frac{\partial}{\partial \alpha_{n}}\right] \ln Z_{\bar{\alpha}_{5}} \\
\gamma_{\alpha_{6}}(a) & =-\left[1+\alpha_{6} \frac{\partial}{\partial \alpha_{6}} \ln Z_{\alpha_{6}}\right]^{-1}\left[\beta(a) \frac{\partial}{\partial a}+\sum_{n^{\prime}} \alpha_{n} \gamma_{\alpha_{n}}(a) \frac{\partial}{\partial \alpha_{n}}\right] \ln Z_{\alpha_{6}}
\end{aligned}
$$

where $\sum_{n^{\prime}}$ means a sum over 1 to 6 but where the term corresponding to the parameter associated with the left hand side is omitted. We have defined $a=g^{2} /\left(16 \pi^{2}\right)$. Moreover, $\phi \in\left\{A, A^{i}, \bar{c} c, \bar{c}^{i} c^{i}, \bar{c}^{i} c, \bar{c} c^{i}, \psi\right\}$ where we now use the notation that a superscript $i$ on a field indicates that it is a diagonal object. We have chosen to define the anomalous dimensions of the ghost and anti-ghost renormalization constants in both sectors in a non-standard way. This is because in the general interpolating gauge to extract individual anomalous dimensions for each field itself entails making an extra assumption. So, for example, $Z_{\bar{c} c}=Z_{\bar{c}}^{\frac{1}{2}} Z_{c}^{\frac{1}{2}}$. We also note that our convention for defining the relation between the bare and renormalized parameters for $\alpha_{1}$ and $\alpha_{3}$ is

$$
\alpha_{1 \mathrm{o}}=Z_{\alpha_{1}}^{-1} Z_{A} \alpha_{1}, \alpha_{3 \mathrm{o}}=Z_{\alpha_{3}}^{-1} \tilde{Z}_{A} \alpha_{3}
$$

where o denotes the bare quantity.

We now present the actual details of our renormalization. In order to extract all the renormalization constants we have had to select a particular set of Green's functions to renormalize. Since the order in which we consider the particular interactions is crucial, it is worth refering to the actual Feynman rules given in the Appendix A. First, applying the Mincer algorithm, [18, 19, to all the two-point functions we have already discussed, it is straightforward to extract the wave function renormalization constants and those for $\alpha_{1}$ and $\alpha_{3}$ and encode them in the anomalous dimensions as

$$
\begin{gathered}
\gamma_{A}(a)=\frac{1}{6 N_{A}^{o}}\left[N_{A}^{o}\left(C_{A}\left(3 \alpha_{1}-13\right)+8 T_{F} N_{f}\right)\right. \\
\left.+N_{A}^{d}\left(C_{A}\left(-3 \alpha_{1}-9 \alpha_{2}+3 \alpha_{3}-3 \alpha_{2} \alpha_{3}\right)\right)\right] a+O\left(a^{2}\right), \\
\gamma_{A^{i}}(a)=\frac{1}{6}\left[C_{A}\left(3 \alpha_{1} \alpha_{2}+3 \alpha_{1}+9 \alpha_{2}-13\right)+8 T_{F} N_{f}\right] a+O\left(a^{2}\right) \\
\gamma_{\alpha_{1}}(a)=\frac{1}{12 \alpha_{1} N_{A}^{o}}\left[N_{A}^{o}\left(C_{A}\left(-6 \alpha_{1}^{2}-6 \alpha_{1} \alpha_{4}+26 \alpha_{1}-3 \alpha_{4}^{2}\right)-16 \alpha_{1} T_{F} N_{f}\right)\right. \\
+N_{A}^{d} C_{A}\left(\begin{array}{r}
18 \alpha_{1}^{2} \alpha_{2}-12 \alpha_{1}^{2} \bar{\alpha}_{5}+18 \alpha_{1}^{2}-6 \alpha_{1} \alpha_{2}^{2} \alpha_{3}-18 \alpha_{1} \alpha_{2}^{2} \\
-12 \alpha_{1} \alpha_{2} \alpha_{3}+6 \alpha_{1} \alpha_{2} \alpha_{6}+18 \alpha_{1} \alpha_{2}-6 \alpha_{1} \alpha_{3} \\
\left.\left.+12 \alpha_{1} \alpha_{4}-12 \alpha_{1} \bar{\alpha}_{5} \alpha_{6}+6 \alpha_{1} \alpha_{6}-36 \alpha_{2}^{2}+6 \alpha_{4}^{2}\right)\right] a
\end{array}\right.
\end{gathered}
$$




$$
\begin{aligned}
& +O\left(a^{2}\right) \\
\gamma_{\alpha_{3}}(a)= & \frac{1}{12 \alpha_{3}}\left[C_{A}\left(-6 \alpha_{1} \alpha_{2} \alpha_{3}-6 \alpha_{1} \alpha_{3}-6 \alpha_{2} \alpha_{3} \alpha_{6}-18 \alpha_{2} \alpha_{3}-6 \alpha_{3} \alpha_{6}+26 \alpha_{3}-3 \alpha_{6}^{2}\right)\right. \\
\quad & \left.\quad-16 \alpha_{3} T_{F} N_{f}\right] a+O\left(a^{2}\right) \\
\gamma_{\bar{c} c}(a)= & \frac{1}{4 N_{A}^{o}}\left[N_{A}^{o} C_{A}\left(\alpha_{1}-3\right)\right. \\
\quad & \quad+N_{A}^{d} C_{A}\left(3 \alpha_{1} \alpha_{2}-2 \alpha_{1} \bar{\alpha}_{5}+\alpha_{1}+\alpha_{2}^{2} \alpha_{3}-3 \alpha_{2}^{2}-2 \alpha_{2} \alpha_{3}\right. \\
\quad & \left.\left.+\alpha_{2} \alpha_{6}+3 \alpha_{2}+\alpha_{3}+\alpha_{6}\right)\right] a+O\left(a^{2}\right) \\
\gamma_{\bar{c}^{i} c^{i}}(a)= & \frac{C_{A}}{4}\left[-\alpha_{1} \alpha_{2}+2 \alpha_{1} \bar{\alpha}_{5}-\alpha_{1}-\alpha_{2} \alpha_{6}-3 \alpha_{2}-\alpha_{6}-3\right] a+O\left(a^{2}\right) \\
\gamma_{\psi}(a)= & \frac{1}{N_{F}}\left[N_{A}^{o} T_{F}\left(\alpha_{1}-\alpha_{3}\right)+N_{F} C_{F} \alpha_{3}\right] a+O\left(a^{2}\right)
\end{aligned}
$$

where $N_{f}$ is the number of quarks. Due to our particular definition of the renormalization of the gauge parameters $\alpha_{1}$ and $\alpha_{3}$ we note that for completeness their anomalous dimensions are

$$
\begin{gathered}
\gamma_{A}(a)+\gamma_{\alpha_{1}}(a)=\frac{C_{A}}{4 \alpha_{1} N_{A}^{o}}[ \\
\quad N_{A}^{d}\left(6 \alpha_{1}^{2} \alpha_{2}-4 \alpha_{1}^{2} \bar{\alpha}_{5}+4 \alpha_{1}^{2}-2 \alpha_{1} \alpha_{2}^{2} \alpha_{3}-6 \alpha_{1} \alpha_{2}^{2}-6 \alpha_{1} \alpha_{2} \alpha_{3}\right. \\
\left.+2 \alpha_{1} \alpha_{2} \alpha_{6}+4 \alpha_{1} \alpha_{4}-4 \alpha_{1} \bar{\alpha}_{5} \alpha_{6}+2 \alpha_{1} \alpha_{6}-12 \alpha_{2}^{2}+2 \alpha_{4}^{2}\right) \\
\left.+N_{A}^{o}\left(-2 \alpha_{1} \alpha_{4}-\alpha_{4}^{2}\right)\right] a+O\left(a^{2}\right) \\
\gamma_{A^{i}}(a)+\gamma_{\alpha_{3}}(a)=-\frac{\alpha_{6} C_{A}}{4 \alpha_{3}}\left[2 \alpha_{2} \alpha_{3}+2 \alpha_{3}+\alpha_{6}\right] a+O\left(a^{2}\right)
\end{gathered}
$$

The next stage is to consider the vertex renormalization. Equipped with the diagonal and offdiagonal gluon and quark anomalous dimensions we have renormalized the triple off-diagonal gluon, quark off-diagonal gluon and quark diagional gluon three-point functions and verified that the correct one loop QCD $\beta$-function of [26] emerges, independent of all six gauge fixing parameters, as

$$
\beta(a)=-\left[\frac{11}{3} C_{A}-\frac{4}{3} T_{F} N_{f}\right] a^{2}+O\left(a^{3}\right) .
$$

This is a useful check on our procedure since it is known that the $\beta$-function is gauge independent in mass independent renormalization schemes. For the four remaining renormalization constants those for $\alpha_{2}$ and $\alpha_{4}$ are the easier to extract. From an examination of the Feynman rules then considering the respective vertices $\left\langle A^{i} A^{i} \bar{c} c\right\rangle$ and $\langle A \bar{c} c\rangle$ each parameter appears as a factor and therefore their renormalization can be extracted by the method of [25] given that $Z_{\bar{c} c}$ has been determined from the off-diagonal ghost two-point function and $Z_{g}$ has first been established as the usual value. In this instance for the three-point function the momentum flow has been carefully chosen so that there is no external momentum flowing into the $\bar{c}$ leg. We find

$$
\begin{aligned}
& \gamma_{\alpha_{2}}(a)=\frac{C_{A}}{8 \alpha_{2} N_{A}^{o}}[ N_{A}^{o}\left(2 \alpha_{1} \alpha_{2}^{2}+2 \alpha_{1} \alpha_{2}+6 \alpha_{2}^{2}+6 \alpha_{2}\right) \\
&+N_{A}^{d}\left(6 \alpha_{1} \alpha_{2}^{2}-2 \alpha_{1} \alpha_{2} \bar{\alpha}_{5}+8 \alpha_{1} \alpha_{2}-2 \alpha_{1} \bar{\alpha}_{5}+2 \alpha_{1}\right. \\
&\left.\left.-6 \alpha_{2}^{3}+\alpha_{2}^{2} \alpha_{6}+2 \alpha_{2} \alpha_{6}+6 \alpha_{2}+\alpha_{6}\right)\right] a+O\left(a^{2}\right) \\
& \gamma_{\alpha_{4}}(a)=\frac{1}{12 \alpha_{4} N_{A}^{o}}\left[N_{A}^{o}\left(C_{A}\left(3 \alpha_{4}^{2}+26 \alpha_{4}\right)-16 \alpha_{4} T_{F} N_{f}\right)\right.
\end{aligned}
$$




$$
\begin{aligned}
+N_{A}^{d} C_{A}( & 18 \alpha_{1} \alpha_{2} \alpha_{4}-12 \alpha_{1} \alpha_{2} \alpha_{6}+12 \alpha_{1} \bar{\alpha}_{5} \alpha_{6}-12 \alpha_{1} \alpha_{6}-18 \alpha_{2}^{2} \alpha_{4} \\
& \left.\left.+36 \alpha_{2}^{2}+6 \alpha_{2} \alpha_{4} \alpha_{6}+18 \alpha_{2} \alpha_{4}-12 \alpha_{4}^{2}+6 \alpha_{4} \alpha_{6}\right)\right] a+O\left(a^{2}\right)
\end{aligned}
$$

To extract $Z_{\alpha_{6}}$ one first requires the renormalization constant $Z_{\bar{c}^{i} c}$ since the vertices where $\alpha_{6}$ appears always involves the combination of fields $\bar{c}^{i}$ and $c$. To do this we consider the vertex $\left\langle A \bar{c}^{i} c\right\rangle$ and choose the momentum flow through the off-diagonal gluon leg to be zero. From the Feynman rule this means that the tree term of the Green's function does not depend on any parameters $\alpha_{i}$ and therefore the only renormalization constant which is undetermined is $Z_{\bar{c}^{i} c}$. Hence,

$$
\begin{aligned}
\gamma_{\bar{c}^{i} c}(a)=\frac{1}{4 N_{A}^{o}} & {\left[N_{A}^{o} C_{A}\left(-\alpha_{1} \alpha_{2}+\alpha_{1}-\alpha_{2} \alpha_{6}-3 \alpha_{2}+\alpha_{4}-\alpha_{6}-3\right)\right.} \\
& \left.+N_{A}^{d} C_{A}\left(-2 \alpha_{1}+2 \alpha_{3}-2 \alpha_{4}+2 \alpha_{6}\right)\right] a+O\left(a^{2}\right) .
\end{aligned}
$$

Equipped with this we can then determine $Z_{\alpha_{6}}$ in two ways. The first is to renormalize the same vertex again but nullify the momentum flow through the $\bar{c}^{i}$ leg which leaves a tree term involving the as yet undetermined $Z_{\alpha_{6}}$. An alternative way is to consider the $\left\langle A A^{i} \bar{c}^{i} c\right\rangle$ vertex given the diagonal and off-diagonal gluon wave function renormalizations and $Z_{\alpha_{2}}$ have already been determined. We have considered both and deduced the same renormalization constant for $Z_{\alpha_{6}}$ for both Green's functions which is a useful check. We found

$$
\begin{gathered}
\gamma_{\alpha_{6}}(a)=\frac{1}{12 N_{A}^{o}}\left[N_{A}^{o}\left(C_{A}\left(-3 \alpha_{1} \alpha_{2}-3 \alpha_{2} \alpha_{6}-9 \alpha_{2}+6 \alpha_{4}-3 \alpha_{6}+26\right)-16 T_{F} N_{f}\right)\right. \\
+N_{A}^{d} C_{A}\left(9 \alpha_{1} \alpha_{2}+6 \alpha_{1} \bar{\alpha}_{5}-9 \alpha_{1}+3 \alpha_{2}^{2} \alpha_{3}-9 \alpha_{2}^{2}+6 \alpha_{2} \alpha_{3}+9 \alpha_{2} \alpha_{6}\right. \\
\left.\left.+9 \alpha_{2}+3 \alpha_{3}-12 \alpha_{4}+9 \alpha_{6}\right)\right] a+O\left(a^{2}\right) .
\end{gathered}
$$

Finally, we follow a similar procedure to extract $Z_{\bar{\alpha}_{5}}$. However, given we have just found $Z_{\alpha_{6}}$ by considering the vertex $\left\langle\bar{c} c \bar{c}^{i} c^{i}\right\rangle$ then $Z_{\bar{\alpha}_{5}}$ can be extracting immediately given that the wave function renormalization for this vertex can be written into the already determined combination $Z_{\bar{c} c}$ and $Z_{\bar{c}^{i} c^{i}}$. This gives

$$
\begin{aligned}
\gamma_{\bar{\alpha}_{5}}(a)=\frac{C_{A}}{4 \alpha_{1} N_{A}^{o}}[ & N_{A}^{o}\left(2 \alpha_{1}^{2} \bar{\alpha}_{5}-2 \alpha_{1}^{2}+\alpha_{4}^{2}\right) \\
& \left.+N_{A}^{d}\left(-4 \alpha_{1} \alpha_{2} \alpha_{6}+4 \alpha_{1} \bar{\alpha}_{5} \alpha_{6}-4 \alpha_{1} \alpha_{6}+12 \alpha_{2}^{2}-2 \alpha_{4}^{2}\right)\right] a+O\left(a^{2}\right) .
\end{aligned}
$$

However, to obtain the complete set of renormalization constants we still need to deduce $Z_{\bar{c} c^{i}}$. Again this can be extracted from two vertices which are $\left\langle A \bar{c} c^{i}\right\rangle$ and $\left\langle A A^{i} \bar{c} c^{i}\right\rangle$ where there is no momentum flowing through the $c^{i}$ leg in the former case. We have renormalized both Green's functions to ensure that the same renormalization constant emerges and found in both cases

$$
\begin{aligned}
\gamma_{\bar{c} c^{i}}(a)=\frac{1}{4 N_{A}^{o}}[ & N_{A}^{o} C_{A}\left(2 \alpha_{1} \bar{\alpha}_{5}-\alpha_{1}-\alpha_{4}-3\right) \\
& +N_{A}^{d} C_{A}\left(3 \alpha_{1} \alpha_{2}-2 \alpha_{1} \bar{\alpha}_{5}+3 \alpha_{1}+\alpha_{2}^{2} \alpha_{3}-3 \alpha_{2}^{2}-2 \alpha_{2} \alpha_{3}\right. \\
& \left.\left.\quad+\alpha_{2} \alpha_{6}+3 \alpha_{2}-\alpha_{3}+2 \alpha_{4}-\alpha_{6}\right)\right] a+O\left(a^{2}\right),
\end{aligned}
$$


which is a useful internal check.

Having produced the anomalous dimensions for the general interpolating gauge we are now in a position to take the various limits of the four special cases discussed above in order to check with the corresponding known anomalous dimensions for these gauges. In comparing with known anomalous dimensions any agreement we indicate is for all the anomalous dimensions except for the interpolating parameters $\alpha_{2}, \alpha_{4}, \bar{\alpha}_{5}$ and $\alpha_{6}$. For the linear covariant gauge case we have

$$
\begin{aligned}
\gamma_{A}(a) & =\gamma_{A^{i}}(a)=\frac{1}{6}\left(C_{A}(3 \alpha-13)+8 T_{F} N_{f}\right) a+O\left(a^{2}\right) \\
\gamma_{\alpha_{1}}(a) & =\gamma_{\alpha_{3}}(a)=-\frac{1}{6}\left(C_{A}(3 \alpha-13)+8 T_{F} N_{f}\right) a+O\left(a^{2}\right) \\
\gamma_{\alpha_{2}}(a) & =\frac{C_{A}}{4 N_{A}^{o}}\left[N_{A}^{o}(\alpha+3)+3 N_{A}^{d}(\alpha+1)\right] a+O\left(a^{2}\right) \\
\gamma_{\alpha_{4}}(a) & =\gamma_{\alpha_{6}}(a)=\frac{1}{6}\left(13 C_{A}-8 T_{F} N_{f}\right) a+O\left(a^{2}\right) \\
\gamma_{\bar{\alpha}_{5}}(a) & =O\left(a^{2}\right) \\
\gamma_{\bar{c} c}(a) & =\gamma_{\bar{c}^{i} c^{i}}(a)=\gamma_{\bar{c} c^{i}}(a)=\gamma_{\bar{c}^{i} c}(a)=\frac{(\alpha-3) C_{A}}{4} a+O\left(a^{2}\right), \\
\gamma_{\psi}(a) & =\alpha C_{F} a+O\left(a^{2}\right)
\end{aligned}
$$

where for $\gamma_{\alpha_{4}}(a)$ we have taken the limit $\alpha_{2} \rightarrow 0$ before setting $\alpha_{4}=0$. These expressions are in agreement with the well established linear covariant gauge anomalous dimensions. For the Curci-Ferrari gauge we have

$$
\begin{aligned}
\gamma_{A}(a) & =\gamma_{A^{i}}(a)=\frac{1}{6}\left(C_{A}(3 \alpha-13)+8 T_{F} N_{f}\right) a+O\left(a^{2}\right) \\
\gamma_{\alpha_{1}}(a) & =\gamma_{\alpha_{3}}(a)=\gamma_{\alpha_{4}}(a)=\gamma_{\alpha_{6}}(a)=-\frac{1}{12}\left(C_{A}(3 \alpha-26)+16 T_{F} N_{f}\right) a+O\left(a^{2}\right) \\
\gamma_{\alpha_{2}}(a) & =\frac{C_{A}}{8 N_{A}^{o}}\left[N_{A}^{o}(2 \alpha+6)+N_{A}^{d}(5 \alpha+6)\right] a+O\left(a^{2}\right), \\
\gamma_{\bar{\alpha}_{5}}(a) & =O\left(a^{2}\right) \\
\gamma_{\bar{c} c}(a) & =\gamma_{\bar{c}^{i} c^{i}}(a)=\gamma_{\bar{c} c^{i}}(a)=\gamma_{\bar{c}^{i} c}(a)=\frac{(\alpha-3) C_{A}}{4} a+O\left(a^{2}\right), \\
\gamma_{\psi}(a) & =\alpha C_{F} a+O\left(a^{2}\right),
\end{aligned}
$$

which are also in agreement with [6, 27, 28, 29]. The equality of the four ghost wave function anomalous dimensions in this and the previous case is consistent with each of the individual wave function ghost anomalous dimensions being equal and equivalent to the value given. For the first of the MAG cases taking the limits with the values in Table 3 we find

$$
\begin{aligned}
\gamma_{A}(a) & =\frac{1}{6 N_{A}^{o}}\left[N_{A}^{o}\left(C_{A}(3 \alpha-13)+8 T_{F} N_{f}\right)+N_{A}^{d} C_{A}(-3 \alpha+9)\right] a+O\left(a^{2}\right), \\
\gamma_{A^{i}}(a) & =-\frac{1}{3}\left(11 C_{A}-4 T_{F} N_{f}\right) a+O\left(a^{2}\right) \\
\gamma_{\alpha_{1}}(a) & =\gamma_{\alpha_{4}}(a)=\frac{1}{12 \alpha N_{A}^{o}}\left[N_{A}^{o}\left(C_{A}\left(-3 \alpha^{2}+26 \alpha\right)-16 \alpha T_{F} N_{f}\right)\right.
\end{aligned}
$$




$$
\begin{aligned}
\left.\quad+N_{A}^{d} C_{A}\left(-6 \alpha^{2}-36 \alpha-36\right)\right] a+O\left(a^{2}\right) \\
\gamma_{\alpha_{2}}(a)=O\left(a^{2}\right) \\
\gamma_{\alpha_{3}}(a)=\frac{1}{3}\left(11 C_{A}-4 T_{F} N_{f}\right) a+O\left(a^{2}\right) \\
\gamma_{\bar{\alpha}_{5}}(a)=\frac{C_{A}}{4 \alpha N_{A}^{o}}\left(N_{A}^{d}\left(12-2 \alpha^{2}\right)-\alpha^{2} N_{A}^{o}\right) a+O\left(a^{2}\right) \\
\gamma_{\alpha_{6}}(a)=\frac{1}{12 N_{A}^{o}}\left[N_{A}^{o}\left(C_{A}(-3 \alpha+35)-16 T_{F} N_{f}\right)+N_{A}^{d} C_{A}(-6 \alpha-18)\right] a+O\left(a^{2}\right) \\
\gamma_{\bar{c} c}(a)=\frac{C_{A}}{4 N_{A}^{o}}\left(N_{A}^{d}(-6-2 \alpha)+N_{A}^{o}(\alpha-3)\right) a+O\left(a^{2}\right) \\
\gamma_{\bar{c}^{i} c^{i}}(a)= \\
\gamma_{\bar{c} c^{i}}(a)=\frac{C_{A}}{4 N_{A}^{o}}\left(N_{A}^{d}(-6-2 \alpha)-3 N_{A}^{o}\right) a+O\left(a^{2}\right) \\
\gamma_{\bar{c}^{i} c}(a)=\frac{\alpha C_{A}}{4} a+O\left(a^{2}\right) \\
\gamma_{\psi}(a)=\frac{\alpha N_{A}^{o} T_{F}}{N_{F}} a+O\left(a^{2}\right)
\end{aligned}
$$

These are in agreement with the one loop arbitrary color group anomalous dimensions recorded in [17. Although unlike the previous two cases the combination of the ghost wave function anomalous dimensions are not equal, their values are consistent with the values given in [17]. Finally, we quote the values for the parameters stated in Table 4 are

$$
\begin{aligned}
\gamma_{A}(a) & =\frac{1}{6 N_{A}^{o}}\left[N_{A}^{o}\left(C_{A}(3 \alpha-13)+8 T_{F} N_{f}\right)-3 \alpha C_{A} N_{A}^{d}\right] a+O\left(a^{2}\right) \\
\gamma_{A^{i}}(a) & =\frac{1}{6}\left((3 \alpha-13) C_{A}+8 T_{F} N_{f}\right) a+O\left(a^{2}\right) \\
\gamma_{\alpha_{1}}(a) & =\gamma_{\alpha_{4}}(a)=\frac{1}{12 N_{A}^{o}}\left[N_{A}^{o}\left(C_{A}(-3 \alpha+26)-16 T_{F} N_{f}\right)+12 \alpha N_{A}^{d} C_{A}\right] a+O\left(a^{2}\right) \\
\gamma_{\alpha_{3}}(a)= & \frac{1}{6}\left((13-3 \alpha) C_{A}-8 T_{F} N_{f}\right) a+O\left(a^{2}\right) \\
\gamma_{\bar{\alpha}_{5}}(a)= & -\frac{\alpha C_{A}}{4 N_{A}^{o}}\left(2 N_{A}^{d}+N_{A}^{o}\right) a+O\left(a^{2}\right) \\
\gamma_{\alpha_{6}}(a)= & \frac{1}{12 N_{A}^{o}}\left[N_{A}^{o}\left(C_{A}(-6 \alpha+26)-16 T_{F} N_{f}\right)+3 \alpha N_{A}^{d} C_{A}\right] a+O\left(a^{2}\right) \\
\gamma_{\bar{c} c}(a)= & \frac{C_{A}}{4 N_{A}^{o}}\left(\alpha N_{A}^{d}+N_{A}^{o}(\alpha-3)\right) a+O\left(a^{2}\right) \\
\gamma_{\bar{c}^{i} c^{i}}(a)= & -\frac{(\alpha+3) C_{A}}{4} a+O\left(a^{2}\right) \\
\gamma_{\bar{c} c^{i}}(a)= & \frac{C_{A}}{4 N_{A}^{o}}\left(\alpha N_{A}^{d}-3 N_{A}^{o}\right) a+O\left(a^{2}\right) \\
\gamma_{\bar{c}^{i} c}(a)= & -\frac{3 C_{A}}{4} a+O\left(a^{2}\right) \\
\gamma_{\psi}(a)= & \frac{\alpha N_{A}^{o} T_{F}}{N_{F}} a+O\left(a^{2}\right)
\end{aligned}
$$


where $\gamma_{\alpha_{2}}(a)$ is singular as $\alpha_{2} \rightarrow 0$. However, this singularity does not affect the anomalous dimensions of the gauge parameters entering the modified maximal Abelian gauge. We regard the results (5.19) as new since as far as we are aware there are no similar computations in this specific gauge.

\section{Conclusion.}

We have constructed a six parameter family of covariant gauge fixings, which allows us to interpolate between various familiar gauges: the Landau, the linear covariant, the Curci-Ferrari and the (modified) maximal Abelian gauges. Using the algebraic renormalization formalism, we have been able to prove the renormalizability of the proposed gauge to any order of perturbation theory. In addition, we have also computed explicitly every anomalous dimension at one-loop order in the $\overline{\mathrm{MS}}$ scheme, confirming that the obtained results reduce to the already known values in the various special cases, if already known. The gauge parameter independence of the gauge beta function was confirmed.

We end by noticing that the concept of an interpolating gauge may also find potential application in lattice numerical simulations. More precisely, requiring that the functional

$$
\mathcal{R}_{k}[A]=(V T)^{-1} \int d^{4} x\left(A_{\mu}^{a} A^{\mu a}+k A_{\mu}^{i} A^{i \mu}\right)
$$

with $k$ the interpolating parameter, attains a minimum with respect to local gauge transformations, leads to the MAG for $k=0$ and to the Landau gauge for $k=1$. This could be useful in order to study the presence, in the MAG, of power corrections in $\frac{1}{q^{2}}$ to the strong coupling constant. Such power corrections are by now quite well established in the Landau gauge, 30, 31, 32, 33. As it turns out that one can interpolate from the Landau gauge to the MAG, one could therefore expect similar corrections in the MAG. One might also hope to obtain some information on the relevance of the Gribov copies in the MAG.

\section{Acknowledgments.}

The Conselho Nacional de Desenvolvimento Científico e Tecnológico (CNPq-Brazil), the Faperj, Fundação de Amparo à Pesquisa do Estado do Rio de Janeiro, the SR2-UERJ and the Coordenação de Aperfeiçoamento de Pessoal de Nível Superior (CAPES) are gratefully acknowledged for financial support.

\section{A Feynman rules.}

In this appendix we list the explicit Feynman rules we used in momentum space. For the propagators we have

$$
\left\langle A_{\mu}^{a}(p) A_{\nu}^{b}(-p)\right\rangle=-\frac{\delta^{a b}}{p^{2}}\left[\eta_{\mu \nu}-\left(1-\alpha_{1}\right) \frac{p_{\mu} p_{\nu}}{p^{2}}\right],
$$




$$
\begin{aligned}
\left\langle A_{\mu}^{i}(p) A_{\nu}^{j}(-p)\right\rangle & =-\frac{\delta^{i j}}{p^{2}}\left[\eta_{\mu \nu}-\left(1-\alpha_{3}\right) \frac{p_{\mu} p_{\nu}}{p^{2}}\right], \\
\left\langle c^{a}(p) \bar{c}^{b}(-p)\right\rangle & =\frac{\delta^{a b}}{p^{2}},\left\langle c^{i}(p) \bar{c}^{j}(-p)\right\rangle=\frac{\delta^{i j}}{p^{2}},\langle\psi(p) \bar{\psi}(-p)\rangle=\frac{\not p}{p^{2}},
\end{aligned}
$$

where $p$ is the momentum. The non-zero 3 - and 4-point vertices are

$$
\begin{aligned}
& \left\langle A_{\mu}^{a}\left(p_{1}\right) \bar{\psi}\left(p_{2}\right) \psi\left(p_{3}\right)\right\rangle=g T^{a} \gamma_{\mu}, \\
& \left\langle A_{\mu}^{i}\left(p_{1}\right) \bar{\psi}\left(p_{2}\right) \psi\left(p_{3}\right)\right\rangle=g T^{i} \gamma_{\mu}, \\
& \left\langle A_{\mu}^{a}\left(p_{1}\right) \bar{c}^{b}\left(p_{2}\right) c^{c}\left(p_{3}\right)\right\rangle=-i g f^{a b c}\left(-\left(1+\frac{\alpha_{4}}{2 \alpha_{1}}\right) p_{1}-p_{3}\right)_{\mu}, \\
& \left\langle A_{\mu}^{a}\left(p_{1}\right) \bar{c}^{b}\left(p_{2}\right) c^{k}\left(p_{3}\right)\right\rangle=-i g f^{a b k}\left(-\bar{\alpha}_{5} p_{1}-\left(1+\alpha_{2}\right) p_{3}\right)_{\mu}, \\
& \left\langle A_{\mu}^{a}\left(p_{1}\right) \bar{c}^{j}\left(p_{2}\right) c^{c}\left(p_{3}\right)\right\rangle=-i g f^{a c j}\left(\left(1+\frac{\alpha_{6}}{2 \alpha_{1}}\right) p_{1}+p_{3}\right)_{\mu}, \\
& \left\langle A_{\mu}^{i}\left(p_{1}\right) \bar{c}^{b}\left(p_{2}\right) c^{c}\left(p_{3}\right)\right\rangle=-i g f^{b c i}\left(-\left(1+\frac{\alpha_{6}}{2 \alpha_{3}}\right) p_{1}-\left(1-\alpha_{2}\right) p_{3}\right)_{\mu}, \\
& \left\langle A_{\mu}^{a}\left(p_{1}\right) A_{\nu}^{b}\left(p_{2}\right) A_{\sigma}^{c}\left(p_{3}\right)\right\rangle=i g f^{a b c}\left(\eta_{\nu \sigma}\left(p_{2}-p_{3}\right)_{\mu}+\eta_{\sigma \mu}\left(p_{3}-p_{1}\right)_{\nu}+\eta_{\mu \nu}\left(p_{1}-p_{2}\right)_{\sigma}\right), \\
& \left\langle A_{\mu}^{a}\left(p_{1}\right) A_{\nu}^{b}\left(p_{2}\right) A_{\sigma}^{k}\left(p_{3}\right)\right\rangle=i g f^{a b k}\left(\eta_{\nu \sigma}\left(p_{2}-\frac{\alpha_{2}}{\alpha_{1}} p_{1}-p_{3}\right)_{\mu}+\eta_{\sigma \mu}\left(p_{3}+\frac{\alpha_{2}}{\alpha_{1}} p_{2}-p_{1}\right)_{\nu}\right. \\
& \left.+\eta_{\mu \nu}\left(p_{1}-p_{2}\right)_{\sigma}\right) \\
& \left\langle A_{\mu}^{a}\left(p_{1}\right) A_{\nu}^{b}\left(p_{2}\right) A_{\sigma}^{c}\left(p_{3}\right) A_{\rho}^{d}\left(p_{4}\right)\right\rangle=g^{2}\left[f_{d}^{a b c d}\left(\eta_{\mu \sigma} \eta_{\nu \rho}-\eta_{\mu \rho} \eta_{\nu \sigma}\right)+f_{d}^{a c b d}\left(\eta_{\mu \nu} \eta_{\sigma \rho}-\eta_{\mu \rho} \eta_{\nu \sigma}\right)\right. \\
& +f_{d}^{a d b c}\left(\eta_{\mu \nu} \eta_{\sigma \rho}-\eta_{\mu \sigma} \eta_{\nu \rho}\right)+f_{o}^{a b c d}\left(\eta_{\mu \sigma} \eta_{\nu \rho}-\eta_{\mu \rho} \eta_{\nu \sigma}\right) \\
& \left.+f_{o}^{a c b d}\left(\eta_{\mu \nu} \eta_{\sigma \rho}-\eta_{\mu \rho} \eta_{\nu \sigma}\right)+f_{o}^{a d b c}\left(\eta_{\mu \nu} \eta_{\sigma \rho}-\eta_{\mu \sigma} \eta_{\nu \rho}\right)\right], \\
& \left\langle A_{\mu}^{a}\left(p_{1}\right) A_{\nu}^{b}\left(p_{2}\right) A_{\sigma}^{c}\left(p_{3}\right) A_{\rho}^{l}\left(p_{4}\right)\right\rangle=-g^{2}\left(f_{o}^{a b c l}\left(-\eta_{\mu \sigma} \eta_{\nu \rho}+\eta_{\mu \rho} \eta_{\nu \sigma}\right)+f_{o}^{a c b l}\left(-\eta_{\mu \nu} \eta_{\sigma \rho}+\eta_{\mu \rho} \eta_{\nu \sigma}\right)\right. \\
& \left.+f_{o}^{a l b c}\left(-\eta_{\mu \nu} \eta_{\sigma \rho}+\eta_{\mu \sigma} \eta_{\nu \rho}\right)\right), \\
& \left\langle A_{\mu}^{a}\left(p_{1}\right) A_{\nu}^{b}\left(p_{2}\right) A_{\sigma}^{k}\left(p_{3}\right) A_{\rho}^{l}\left(p_{4}\right)\right\rangle=-g^{2}\left(f_{o}^{a k b l}\left(-\eta_{\mu \nu} \eta_{\sigma \rho}-\frac{\alpha_{2}^{2}}{\alpha_{1}} \eta_{\mu \sigma} \eta_{\nu \rho}+\eta_{\mu \sigma} \eta_{\nu \rho}+\eta_{\mu \rho} \eta_{\nu \sigma}\right)\right. \\
& \left.+f_{o}^{a l b k}\left(-\eta_{\mu \nu} \eta_{\sigma \rho}+\eta_{\mu \sigma} \eta_{\nu \rho}-\frac{\alpha_{2}^{2}}{\alpha_{1}} \eta_{\mu \rho} \eta_{\nu \sigma}\right)\right), \\
& \left\langle A_{\mu}^{a}\left(p_{1}\right) A_{\nu}^{b}\left(p_{2}\right) \bar{c}^{c}\left(p_{3}\right) c^{d}\left(p_{4}\right)\right\rangle=-g^{2}\left(f_{d}^{a c b d} \alpha_{2} \eta_{\mu \nu}+f_{d}^{b c a d} \alpha_{2} \eta_{\mu \nu}\right), \\
& \left\langle A_{\mu}^{a}\left(p_{1}\right) A_{\nu}^{j}\left(p_{2}\right) \bar{c}^{c}\left(p_{3}\right) c^{d}\left(p_{4}\right)\right\rangle=-g^{2}\left(f_{o}^{a d c j} \alpha_{2} \eta_{\mu \nu}+f_{o}^{a j c d} \frac{\alpha_{2} \alpha_{4}}{2 \alpha_{1}} \eta_{\mu \nu}\right), \\
& \left\langle A_{\mu}^{a}\left(p_{1}\right) A_{\nu}^{j}\left(p_{2}\right) \bar{c}^{c}\left(p_{3}\right) c^{l}\left(p_{4}\right)\right\rangle=-g^{2}\left(f_{o}^{a j c l} \frac{\alpha_{2} \alpha_{5}}{2 \alpha_{1}} \eta_{\mu \nu}+f_{o}^{a l c j} \alpha_{2} \eta_{\mu \nu}\right), \\
& \left\langle A_{\mu}^{a}\left(p_{1}\right) A_{\nu}^{j}\left(p_{2}\right) \bar{c}^{k}\left(p_{3}\right) c^{d}\left(p_{4}\right)\right\rangle=-g^{2}\left(-f_{o}^{a j d k} \frac{\alpha_{2} \alpha_{6}}{2 \alpha_{1}} \eta_{\mu \nu}\right),
\end{aligned}
$$

\footnotetext{
${ }^{\ddagger}$ We note that in 17 the corresponding Feynman rules for the four-point corrections did not all have the correct coupling constant factor of $g^{2}$ and the $\left\langle A A A^{i}\right\rangle$ vertex was inadvertently omitted.
} 


$$
\begin{aligned}
\left\langle A_{\mu}^{i}\left(p_{1}\right) A_{\nu}^{j}\left(p_{2}\right) \bar{c}^{c}\left(p_{3}\right) c^{d}\left(p_{4}\right)\right\rangle= & -g^{2}\left(-f_{o}^{c i d j} \alpha_{2} \eta_{\mu \nu}-f_{o}^{c j d i} \alpha_{2} \eta_{\mu \nu}\right), \\
\left\langle\bar{c}^{a}\left(p_{1}\right) c^{b}\left(p_{2}\right) \bar{c}^{c}\left(p_{3}\right) c^{d}\left(p_{4}\right)\right\rangle= & -g^{2}\left(-\frac{\alpha_{6}^{2}}{4 \alpha_{3}} f_{d}^{a b c d}-\frac{\alpha_{5}}{2} f_{d}^{a c b d}-\frac{\alpha_{6}^{2}}{4 \alpha_{3}} f_{d}^{a d b c}\right. \\
& \left.\quad-\frac{\alpha_{4}^{2}}{4 \alpha_{1}} f_{o}^{a b c d}-\frac{\alpha_{4}}{2} f_{o}^{a c b d}-\frac{\alpha_{4}^{2}}{4 \alpha_{1}} f_{o}^{a d b c}\right), \\
\left\langle\bar{c}^{a}\left(p_{1}\right) c^{b}\left(p_{2}\right) \bar{c}^{c}\left(p_{3}\right) c^{l}\left(p_{4}\right)\right\rangle= & -g^{2}\left(-\frac{\alpha_{4} \alpha_{5}}{4 \alpha_{1}} f_{o}^{a b c l}-\frac{\alpha_{4}}{2} f_{o}^{a c b l}-\frac{\alpha_{4} \alpha_{5}}{4 \alpha_{1}} f_{o}^{a l b c}\right), \\
\left\langle\bar{c}^{a}\left(p_{1}\right) c^{b}\left(p_{2}\right) \bar{c}^{k}\left(p_{3}\right) c^{d}\left(p_{4}\right)\right\rangle= & -g^{2}\left(\frac{\alpha_{4} \alpha_{6}}{4 \alpha_{1}} f_{o}^{a b d k}-\frac{\alpha_{4} \alpha_{6}}{4 \alpha_{1}} f_{o}^{a d b k}-\frac{\alpha_{6}}{2} f_{o}^{a k b d}\right), \\
\left\langle\bar{c}^{a}\left(p_{1}\right) c^{b}\left(p_{2}\right) \bar{c}^{k}\left(p_{3}\right) c^{l}\left(p_{4}\right)\right\rangle= & -g^{2}\left(-\frac{\alpha_{6}}{2} f_{o}^{a k b l}-\frac{\alpha_{5} \alpha_{6}}{4 \alpha_{1}} f_{o}^{a l b k}\right), \\
\left\langle\bar{c}^{a}\left(p_{1}\right) c^{j}\left(p_{2}\right) \bar{c}^{c}\left(p_{3}\right) c^{l}\left(p_{4}\right)\right\rangle= & 0, \\
\left\langle\bar{c}^{i}\left(p_{1}\right) c^{b}\left(p_{2}\right) \bar{c}^{k}\left(p_{3}\right) c^{d}\left(p_{4}\right)\right\rangle= & 0,
\end{aligned}
$$

where the momentum, $p_{i}$, flow into each vertex and we have introduced the more compact notation, [17,

$$
f_{d}^{A B C D}=f^{i A B} f^{i C D}, f_{o}^{A B C D}=f^{e A B} f^{e C D},
$$

for the four-point interactions with $i, j, k$ and $l$ denoting indices of objects in the group centre. The Feynman rules for the remaining possible combinations of diagonal and off-diagonal fields are trivially zero since they would generate group factors involving $f^{i j c}$ or $f^{i j k}$ which are both zero from the Lie algebra.

\section{References.}

[1] O. Piguet, G. Pollak and M. Schweda, Nucl. Phys. B328 (1989), 527.

[2] L. Baulieu and D. Zwanziger, Nucl. Phys. B548 (1999), 527.

[3] C. S. Fischer and D. Zwanziger, hep-ph/0504244.

[4] D. Dudal, J.A. Gracey, V.E.R. Lemes, M.S. Sarandy, R.F. Sobreiro, S.P. Sorella and H. Verschelde, Phys. Rev. D70 (2004), 114038.

[5] D. Dudal, H. Verschelde, J.A. Gracey, V.E.R. Lemes, M.S. Sarandy, R.F. Sobreiro and S.P. Sorella, JHEP 0401 (2004), 044.

[6] G. Curci and R. Ferrari, Nuovo Cim. A32 (1976), 151.

[7] R. Delbourgo and P.D. Jarvis, J. Phys. A15 (1982), 611.

[8] L. Baulieu and J. Thierry-Mieg, Nucl. Phys. B197 (1982), 477.

[9] H. Min, T. Lee and P.Y. Pac, Phys. Rev. D32 (1985), 440.

[10] A.R. Fazio, V.E.R. Lemes, M.S. Sarandy and S.P. Sorella, Phys. Rev. D64 (2001), 085003. 
[11] K. Amemiya and H. Suganuma, Phys. Rev. D60 (1999), 114509.

[12] V.G. Bornyakov, M.N. Chernodub, F.V. Gubarev, S.M. Morozov and M.I. Polikarpov, Phys. Lett. B559 (2003), 214.

[13] D. Dudal, V.E.R. Lemes, M.S. Sarandy, S.P. Sorella and M. Picariello, JHEP 0212 (2002), 008.

[14] O. Piguet and S.P. Sorella, Lect. Notes Phys. M28 (1995), 1.

[15] D. Dudal, H. Verschelde, V.E.R. Lemes, M.S. Sarandy, R.F. Sobreiro, S.P. Sorella, M. Picariello and J.A. Gracey, Phys. Lett. B569 (2003), 57.

[16] D. Dudal, H. Verschelde, V.E.R. Lemes, M.S. Sarandy, R.F. Sobreiro, S.P. Sorella and J.A. Gracey, Phys. Lett. B574 (2003), 325.

[17] J.A. Gracey, JHEP 0504 (2005), 012.

[18] S.G. Gorishny, S.A. Larin, L.R. Surguladze and F.K. Tkachov, Comput. Phys. Commun. 55 (1989), 381.

[19] S.A. Larin, F.V. Tkachov and J.A.M. Vermaseren, "The Form version of Mincer", NIKHEFH-91-18.

[20] J.A.M. Vermaseren, math-ph/0010025.

[21] P. Nogueira, J. Comput. Phys. 105 (1993), 279.

[22] M. Misiak and M. Münz, Phys. Lett. B344 (1995), 308.

[23] K.G. Chetyrkin, M. Misiak and M. Münz, Nucl. Phys. B518 (1998), 473.

[24] S. Weinberg, Phys. Rev. 118 (1960), 838.

[25] S.A. Larin, Phys. Lett. B303 (1993), 113.

[26] D.J. Gross and F.J. Wilczek, Phys. Rev. Lett. 30 (1973), 1343; H.D. Politzer, Phys. Rev. Lett. 30 (1973), 1346.

[27] J. de Boer, K. Skenderis, P. van Nieuwenhuizen and A. Waldron, Phys. Lett. B367 (1996), 175 .

[28] J.A. Gracey, Phys. Lett. B525 (2002), 89.

[29] R.E. Browne and J.A. Gracey, Phys. Lett. B540 (2002), 68.

[30] P. Boucaud, A. Le Yaouanc, J. P. Leroy, J. Micheli, O. Pene and J. Rodriguez-Quintero, Phys. Rev. D63 (2001), 114003.

[31] E. Ruiz Arriola, P. O. Bowman and W. Broniowski, Phys. Rev. D70 (2004), 097505.

[32] S. Furui and H. Nakajima, hep-lat/0503029.

[33] P. Boucaud, F. de Soto, J.P. Leroy, A. Le Yaouanc, J. Micheli, H. Moutarde, O. Pene and J. Rodriguez-Quintero, hep-lat/0504017 